\title{
Design of punching shear for prestressed slabs with unbonded tendons on internal columns
}

\section{Dimensionamento à punção de lajes protendidas com cordoalhas engraxadas em apoios internos}

L. A. R. LUCHI a lorenzo@rl.eng.br

J. C. C. LEITE JR. a junior@civix.eng.br

\begin{abstract}
This paper is related to the punching shear in prestressed slabs with unbonded tendons for interior columns calculated by the codes ABNT NBR 6118:2007, ABNT NBR 6118:2014, EN 1992-1-1:2004 e ACI 318-11. To calculate the punching shear resistance the formulations of the NBR 6118:07, effective until April/2014, did not consider the compression of the concrete in the plane of the slab, due to prestressing. Just the inclined components of some tendons were considered for total load applied relief, but this fact did not generate a significant difference, compared to reinforced concrete, because the inclination angle is very close to zero. The American and European provisions consider a portion related to the compression of the concrete in the plane of the slab. Differences in the results obtained by the four design codes will be exposed, showing that the EC2:04 and the NBR6118:14 achieved the best results.
\end{abstract}

Keywords: slab, punching shear, post-tensioning, codes.

\section{Resumo}

Este artigo trata da punção em lajes protendidas com cordoalhas não aderentes na ligação laje-pilar interno calculada através das normas ABNT NBR 6118:2007, ABNT NBR 6118:2014, EN 1992-1-1:2004 e ACI 318-11. Para o dimensionamento à punção, as formulações da NBR 6118:2007, norma que estava vigente até abril de 2014, não levavam em conta a compressão do concreto no plano da laje devido à protensão. Apenas a componente inclinada de uma parte das cordoalhas era considerada para alívio da carga solicitante de cálculo; entretanto, isso não gerava diferença significativa, com relação ao concreto armado, pelo fato de o ângulo de inclinação ser bem próximo de zero. As normas americana e europeia consideram uma parcela referente à compressão do concreto no plano da laje. Serão expostas, portanto, as diferenças de resultados obtidos através dos quatro códigos de dimensionamento, mostrando que o EC2:2004 e a NBR 6118:14 obtiveram os melhores resultados.

Palavras-chave: laje, punção, protensão, normas. 


\section{Introduction}

\subsection{Justification and motivation}

The motivation for this article is due to the fact that the Brazilian, European and American codes have different formulations for calculating the punching shear in prestressed slabs, generating different results from the same input data. In addition, the existing literature shows that the codes results in a conservative design, compared with real of tests on prestressed slabs with unbonded tendons.

For punching shear design, formulations of the NBR 6118: 2007 standard that was in effect until April 2014, do not consider the concrete compression in the slab plane, due to prestressing. Just the inclined component of some tendons was considered to relief the requesting load; however, it did not generate significant difference compared with reinforced concrete, because the inclination angle is very close to zero. The American and European standards consider a portion related to concrete compression in the slab plane. Thus, in order to obtain less conservative results, ABNT revised the formulation to design punching shear in the new NBR 6118: 2014 by inserting a portion related to compression in the slab plane, due to prestressing as Eurocode 2 suggests.

It is known that structural designs should be safe, but it is part of the engineering function to always improve the methods of design and execution to obtain more viable and economic results. Therefore, the results of this paper can show the influence of compressed concrete by the use of prestressing with greased tendons.

\subsection{Objectives}

The purpose of this article is to compare the formulations for sizing punching shear provided by NBR 6118: 2007, NBR 6118 : 2014, EN 1992-1-1: 2004 and ACl 318-11. Several dimensioning will be performed by changing load levels, sections of pillars, concrete strength and reinforcement ratio, in order to observe the difference in results between the standards, according to these variables.

\section{Dimensioning standards}

\subsection{Brazilian standard (NBR 6118)}

\subsubsection{Design model}

The design model corresponds to checking the shear in two or more critical surfaces. In the first critical surface (section $\mathrm{C}$ ), the diagonal compression strength of the concrete should be checked by shear stress. In the second critical surface (section $C^{\prime}$ ), at a distance of $2 d$ of the column or concentrated load, should be verified punching shear resistance relating to diagonal tension. This verification should also be made for a shear stress in the C' section. A third critical surface (section C"), should be checked only when using shear reinforcement.

\subsubsection{Internal column with symmetrical loading}

For symmetrical loads:

$\tau_{S d}=\frac{F_{S d}}{u d}$

where:

$d=\left(d_{x}+d_{y}\right) / 2$

where:

$d$ is the effective height of the slab along the critical section C'. $d_{x}$ and $d_{y}$ are the effective heights in two orthogonal directions; $u$ is the perimeter of the section C';

$F_{S d}$ is the factored force or concentrated reaction.

\subsubsection{Verification of the resistant strain of diagonal compression of concrete}

This check should be made in the section $\mathrm{C}$, for slabs with or without punching shear reinforcement.

$\tau_{s d} \leq \tau_{R d 2}=0,27 \alpha_{v} f_{c d}$

where:

$\alpha_{v}=\left(1-f_{c k} / 250\right), f_{c k}$ in megapascals.

The value of $\tau_{R d 2}$ may be increased by $20 \%$ for multi-axial states of stress to internal columns when the adjacent spans do not differ more than $50 \%$ and if there are no openings near the column.

\subsubsection{Resistant strain in the critical surface C' in structural elements without punching shear reinforcement}

The resistant strain in the critical surface C' must be calculated, according to NBR 6118:2014, as follows:

$\tau_{S d} \leq \tau_{R d 1}=0,13(1+\sqrt{20 / d})\left(100 \rho f_{c k}\right)^{\frac{1}{3}}+0,10 \sigma_{c p}$

where:

$\rho=\sqrt{\rho_{x} \rho_{y}}$

$d=\left(d_{x}+d_{y}\right) / 2$

where:

$d$ is the effective height of the slab along the critical section C' of the force application area, in centimeters;

$\rho$ is the geometric ratio of flexural reinforcement;

$\rho_{x}$ and $\rho_{y}$ are reinforcement ratios in two orthogonal directions;

$\sigma_{c p}$ is the average compression strain in the slab plane.

NBR 6118:2007 considered the $\tau_{R d 1}$ calculated according to the equation:

$\tau_{S d} \leq \tau_{R d 1}=0,13(1+\sqrt{20 / d})\left(100 \rho f_{c k}\right)^{\frac{1}{3}}$

Therefore, ignoring the compression in the slab plane. 
2.1.5 Resistant strain in the critical surface $C^{\prime}$ in structural elements with punching shear reinforcement

The resistant strain in the critical surface C' must be calculated, according to NBR 6118:2014, as follows:

$\tau_{S d} \leq \tau_{R d 3}=0,10\left(1+\sqrt{\frac{20}{d}}\right)\left(100 \rho f_{c k}\right)^{\frac{1}{3}}+$

$0,10 \sigma_{c p}+1,5 \frac{d}{s_{r}} \frac{A_{s w} f_{y w d} \operatorname{sen} \alpha}{u d}$

where:

$s_{r} \leq 0,75 d$

where:

$s_{r}$ is the radial spacing between the reinforcement punching shear lines, not more than $0,75 \mathrm{~d}$;

$A_{s w}$ is the area of punching shear reinforcement in a full section parallel to C';

$\alpha$ is the inclination angle between the axis of the punching shear reinforcement and the plane of the slab;

$u$ is the critical perimeter;

$f_{y w d}$ is the factored resistance of the punching shear reinforcement, not greater than $300 \mathrm{MPa}$ to connectors or $250 \mathrm{MPa}$ to stirrups (CA$50 / 60$ ). For slabs with thickness greater than $15 \mathrm{~cm}$, these values can be increased from linear interpolation.

NBR 6118:2007 considered the $\tau_{R d 3}$ calculated according to the equation:

$\tau_{S d} \leq \tau_{R d 3}=0,10\left(1+\sqrt{\frac{20}{d}}\right)\left(100 \rho f_{c k}\right)^{\frac{1}{3}}+$

$1,5 \frac{d}{s_{r}} \frac{A_{s w} f_{y w d} \operatorname{sen} \alpha}{u d}$

Therefore, ignoring the compression in the slab plane

\subsubsection{Definition of the critical surface C"}

When it is necessary to use punching shear reinforcement, it must be extended in parallel sections to C' until an outline C", away $2 d$ from the last section, when reinforcement is no longer necessary $\left(\tau_{S d} \leq \tau_{R d 1}\right)$.

\subsubsection{Verification of prestressed structural elements}

The check should be made as follows:

$\tau_{S d, e f}=\tau_{S d}-\tau_{P d}$

where:

$\tau_{P d}=\frac{\sum\left(P_{k \text { inf }, i} \operatorname{sen} \alpha_{i}\right)}{u d}$

where:

$\tau_{P d}$ is the stress due to the effect of inclined prestressing cables crossing the section considered in a perimeter away $d / 2$ from the column face.
$P_{\text {kinf }, i}$ is the prestressing force un the cable $i$

$\alpha$ is the inclination angle of the cable $i$ relative to slab plane; $u$ is the critical perimeter of the considered section, in which are calculated $\tau_{\mathrm{Sd}, \text { ef }}$ and $\tau_{\mathrm{Sd}}$.

\subsection{American standard (ACl 318)}

\subsubsection{Control perimeters}

The American standard recommends the analysis of stresses in critical sections located at a distance of $d / 2$ ( $d$ is the useful height of the slab), from the face of the columns or concentrated loads. The perimeter of these sections is called effective perimeter $b_{0}$. After determining that perimeter, the resistant strain and actuating strain are compared. If necessary, the slab thickness may be increased or punching shear reinforcement can be added to increase the resistant strain.

\subsubsection{Calculation of actuating strain $\tau_{u}$ in the affective perimeter $b$ 。}

The shear stress actuating on the slab-internal column connection for centered loads, is given by:

$\tau_{u}=\frac{F_{u}}{A_{c}}$

where:

$F_{u}$ is the factored shear force;

$A_{c}$ is the concrete area of the effective critical section.

\subsubsection{Calculation of resistant strain $\tau_{u}$ in the affective perimeter $b$ 。}

- Resistant strain of slab without punching shear reinforcement:

$\tau_{u} \leq \tau_{n}=\tau_{c}$

$\tau$ is the strain related to the concrete, taken as the smallest of the two following values:

$\tau_{c}=0,17\left(1+\frac{2}{\beta}\right) \sqrt{f^{\prime}{ }_{c}} \leq 0,33 \sqrt{f^{\prime}}$

$\tau_{c}=0,083\left(\frac{\alpha_{s} d}{b_{o}}+2\right) \sqrt{f^{\prime}} \leq 0,33 \sqrt{f^{\prime}{ }_{c}}$

where:

$f^{\prime} c$ is the specified compression strengh of concrete, in MPa;

$b_{0}$ is the critical section for shear in slabs;

(11) $\beta$ is the ratio between the largest and the smallest side of the column;

$d$ is the effective height of the slab;

$\alpha_{s}$ is equal to 40 for internal columns.

- Resistant strain of slab with punching shear reinforcement:

$\tau_{u} \leq \tau_{n}=\tau_{c}+\tau_{s} \leq 0,5 \sqrt{f^{\prime}}{ }_{c}$

$\tau_{c}=0,17 \sqrt{f^{\prime}}$ 
where:

$\tau_{c}$ is the strain related to the concrete;

$\tau_{s}$ is the strain related do the reinforcement steel:

$\tau_{s}=\frac{A_{v} f_{y t}(\operatorname{sen} \alpha+\cos \alpha)}{s b_{0}}$

where:

$A_{v}$ is the area of the slab shear reinforcement;

$f_{y t}$ is the yield stress of the punching shear reinforcement steel, in

$\mathrm{MPa}$, less than or equal to $400 \mathrm{MPa}$;

$b_{o}$ is the critical section for shear;

$\alpha$ is the inclination angle between the axis of the punching shear reinforcement and the plane of the slab;

$s$ is the reinforcement spacing, in millimeters.

The punching shear reinforcement must be extended on parallel contours to the edge of the column faces, until a distance equal to $d / 2$ of the last line of reinforcement, the shear stress applied is not larger than $0,17 \sqrt{f_{c}^{\prime}}$.

\subsubsection{Verification for prestressed structural elements}

For prestressed slabs, the shear strength of the concrete can be calculated from the following formulation:

$\tau_{c}=\left(\beta \sqrt{f^{\prime}}+0,30 f_{p c}\right)+\frac{V_{p}}{b_{0} d}$

where:

$f_{c}^{\prime}$ is the specified compression strengh of concrete;

$\beta_{p}$ is the lowest value between 0,29 and $0,083\left(\frac{\alpha_{s} \mathrm{~d}}{\mathrm{~b}_{\mathrm{o}}}+1,5\right)$;
$d$ is the effective height of the slab;

$b_{0}$ is the critical section for shear;

$\alpha_{s}$ is equal to 40 for internal columns;

$f_{p c}$ is the average compression strength of the concrete in both orthogonal directions due to prestressing;

$V_{P}$ is the vertical component of prestressing forces located within the critical section.

It should also take into consideration:

any part of the cross section of the column can not be more than four times the height of the slab next to a discontinuous edge;

- $\mathrm{f}_{\mathrm{c}}$, value can not be greater than $33,64 \mathrm{MPa}$;

in each direction, $f_{p c}$ can not be less than $0,9 \mathrm{MPa}$ or greater than $3,5 \mathrm{MPa}$.

\subsection{European standard (EC2)}

The European Standard recommends that punching shear analysis must be first made on the perimeter of the face (perimeter $u_{0}$ ) and then in a perimeter away the $2 \mathrm{~d}$ of the face or concentrated load (perimeter $u_{1}$ ). If the shear reinforcement is needed, another contour must be checked out of the last line of reinforcement, called perimeter $u_{2}$.

\subsubsection{Calculation of applicants strains in control perimeters}

$\tau_{E d}=\beta \frac{F_{E d}}{u_{i} d}$ where:

$F_{E d}$ is the factored shear force;

$d$ is the effective height of the slab;

$u_{i}$ is the considered control perimeter;

$\beta$ is the factor corresponding to bending moment.

\subsubsection{Calculation of resistant strains in control perimeters}

In the control perimeter $u_{0}$ :

$\tau_{E d} \leq \tau_{R d, \text { máx }}=0,5_{v_{f_{c d}}}$

where:

$v=0,6(1-\mathrm{fck} / 250)$;

$f_{c d}$ is the fatored compression strengh of concrete (MPa).

- In the control perimeter $u_{i}$ :

a) For elements without punching shear reinforcement:

$\tau_{\mathrm{Ed}} \leq \tau_{\mathrm{Rd}, \mathrm{c}}=0,12 \mathrm{k}\left(100 \rho_{1} \mathrm{f}_{\mathrm{ck}}\right)^{\frac{1}{3}} \geq 0,035(\mathrm{k})^{\frac{3}{2}}\left(\mathrm{f}_{\mathrm{ck}}\right)^{\frac{1}{2}}$

where:

$k=1+\sqrt{\frac{200}{d}} \leq 2$

$\rho_{1}$ is the calculated average ratio of reinforcement considering the width of the column adding $3 d$ for each face.

$f_{c k}$ in $\mathrm{MPa}$ and $d$ in millimeters.

b) For elements with punching shear reinforcement:

$\tau_{\text {Ed }} \leq \tau_{R d, c s}=0,75 \tau_{R d, c}+1,5\left(d / s_{r}\right) A_{s w} f_{y w d, \text { ef }}\left[1 /\left(u_{1} d\right)\right] \operatorname{sen} \alpha$

where:

$A_{s w}$ is the punching shear reinforcement area in the perimeter considered $\left(\mathrm{mm}^{2}\right)$;

$s_{r}$ is the radial spacing between the reinforcement punching shear lines $(\mathrm{mm})$;

$f_{\text {ywd,ef }}$ is the effective resistance of the reinforcement

$\left(\mathrm{f}_{\mathrm{ywd} \text {,ef }}=250+0,25 \mathrm{~d} \leq \mathrm{f}_{\mathrm{ywd}}\right.$ in MPa);

$d$ is the effective average thickness of the slabs, in both directions $(\mathrm{mm})$;

$\alpha$ is the angle between the shear reinforcement and the slab plane.

\subsubsection{Verification for prestressed slabs}

In the case of prestressed slabs, the concrete strength can be set by the expression:

$\tau_{R d, e f}=\tau_{R d, c}+0,1 \sigma_{c p}$

where:

$\sigma_{c p}$ is the average compression strain in the slab plane.

The vertical component $V_{p}$, resulting from the inclined cables that pass through the perimeter $u_{1}$, can be considered as relief of shear load.

$V_{p}=2\left(n_{x} \cdot P_{p} \cdot \operatorname{sen} \alpha_{x}\right)+2\left(n_{y} \cdot P_{p} \cdot \operatorname{sen} \alpha_{y}\right)$

where:

$n_{x}$ and $n_{y}$ is the number of cables that cross the control section in each direction; 
$\mathrm{P}_{\mathrm{p}}$ is the average prestressing force (at rupture);

$\alpha_{x}$ and $\alpha_{y}$ are the inclination angles of the cables in the boundary of control sections.

\section{Comparative designs and results}

\subsection{Considerations}

For the comparative analysis of shear reinforcement, forces have been considered ranging from $400 \mathrm{kN}$ to $1200 \mathrm{kN}$, increases of $100 \mathrm{kN}$, in slabs with thickness of $18 \mathrm{~cm}$.

By prestressing, compressive strains equal to $1,33 \mathrm{MPa}$ and $2,0 \mathrm{MPa}$ were applied in the plane of the slabs. Also, the resistance of the concrete in the calculations were $30 \mathrm{MPa}, 35 \mathrm{MPa}$ and $40 \mathrm{MPa}$.
It was considered two flexural reinforcement ratios, equal to $0.5 \%$ and $1.5 \%$, and also analyzed different sections for the support columns, equal to $30 \times 30 \mathrm{~cm}, 40 \times 40 \mathrm{~cm}$ and $50 \times 50 \mathrm{~cm}$.

It is noteworthy that the radial spacing between the reinforcement stirrups was $10 \mathrm{~cm}$.

The minimum value of puncture force, $400 \mathrm{kN}$, was taken considering slabs supported on columns modulated with $8 \times 8 \mathrm{~m}$ spans - influence area equal to $64 \mathrm{~m}^{2}$ - and minimal overload of $2 \mathrm{kN} / \mathrm{m}^{2}$. The maximum value, $1200 \mathrm{kN}$, results from an overload of $14 \mathrm{kN} / \mathrm{m}^{2}$, considering the same definition of the slabs.

Unbonded tendons were used with a diameter of $12.7 \mathrm{~mm}$ and steel CP190-RB. The prestressing force was considered $150 \mathrm{kN}$ with losses of $20 \%$. It was considered the tendons of the tilt angle in the region of columns equal to 2.5 degrees.

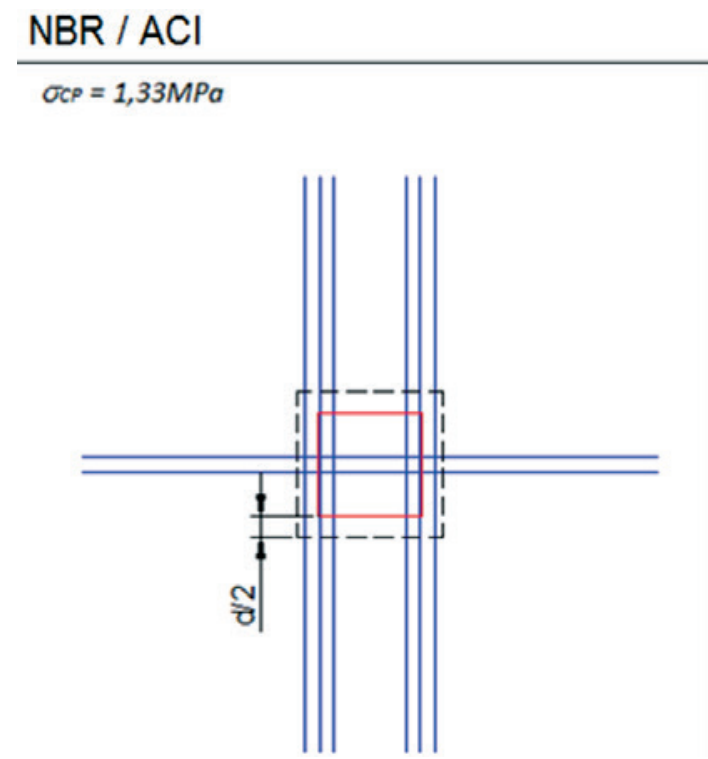

$\sigma c P=2,0 M P a$

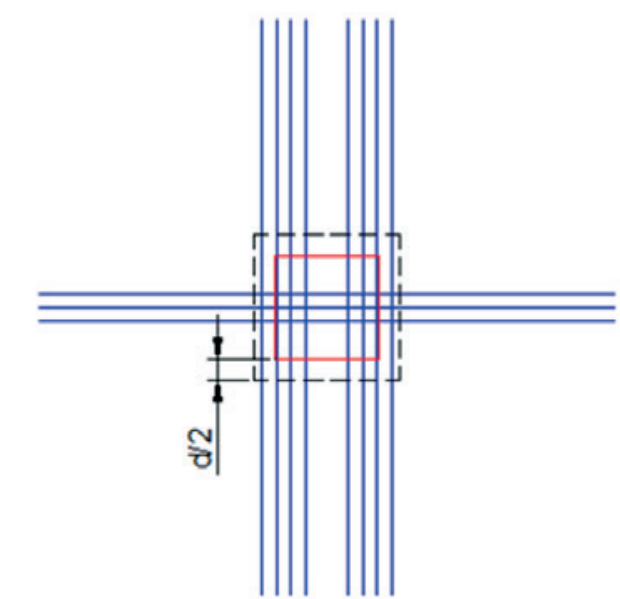

Figure 1

Tendons considered in the calculation of $V_{p}$ by $N B R$ and $\mathrm{ACl}$

Source: Elaborated by the author

\section{EUROCODE}

$$
\sigma c p=1,33 \mathrm{MPa}
$$

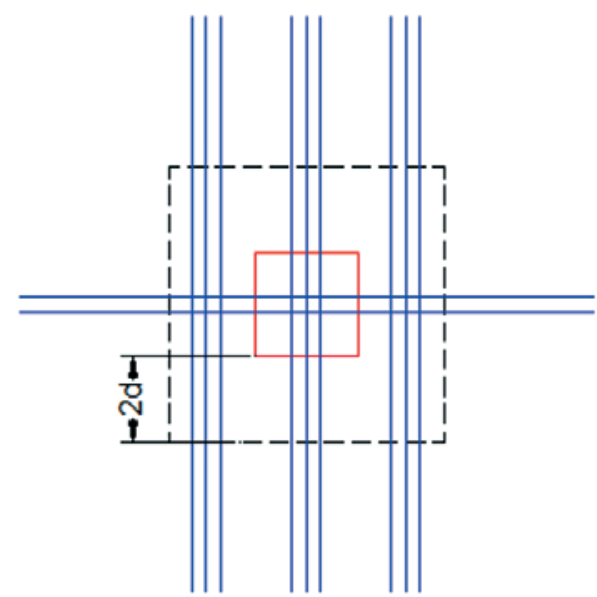

$\sigma c p=2,0 M P a$

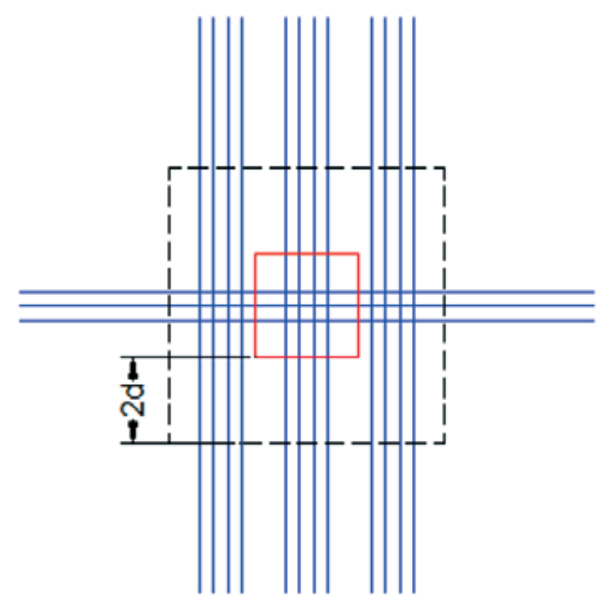

\section{Figure 2}

Tendons considered in the calculation of $V_{p}$ by Eurocode

Source: Elaborated by the author 


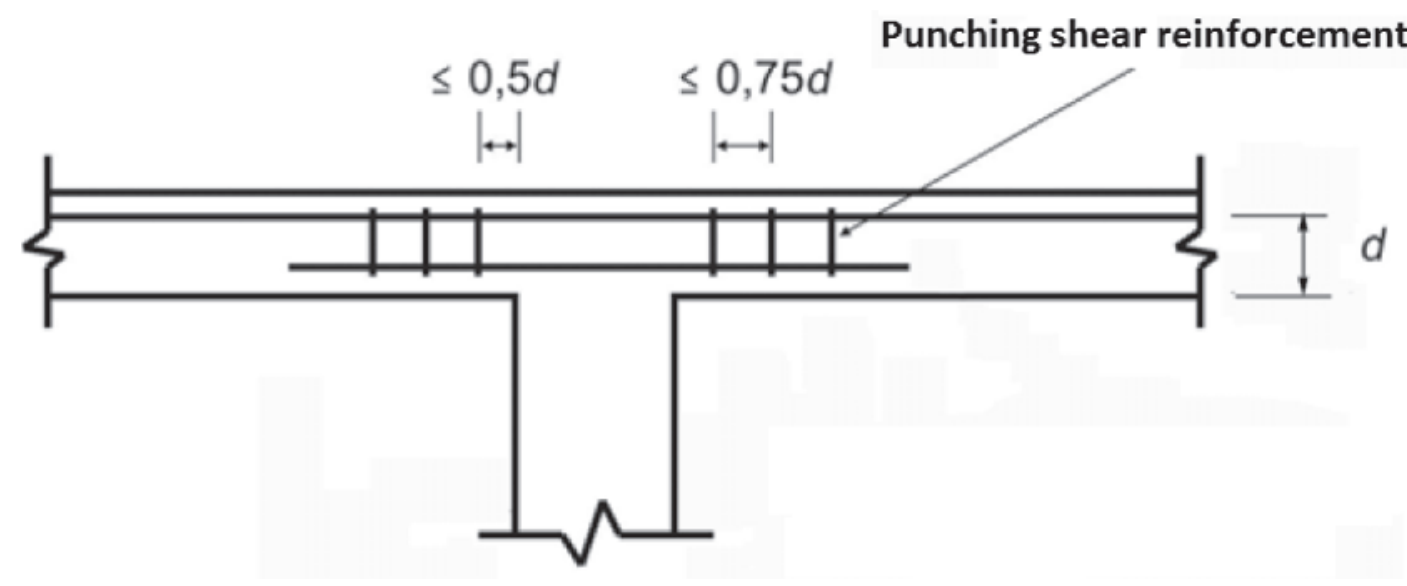

Figure 3

Model using stirrups for shear reinforcement

Source: NBR $6118: 2014$

Figures 1 and 2 show the distribution of the prestressing tendons on the support columns and Figure 3 is the schematic consideration in stirrups adopted in the calculations.

\subsection{Designs of shear reinforcement from the different analyzes}

\subsubsection{Designing from NBR 6118:2007}

Analysis $1 \rightarrow \sigma_{\mathrm{cp}}=1,33 \mathrm{MPa}$ (2 tendons/meter)

Results presented in Table 1.

Analysis $2 \rightarrow \sigma_{c p}=2,0 \mathrm{MPa}$ (3 tendons/meter)

Results presented in Table 2 .

\subsubsection{Designing from NBR 6118:2014}

Analysis $1 \rightarrow \sigma_{\mathrm{cp}}=1,33 \mathrm{MPa}$ (2 tendons/meter)

Results presented in Table 3.

Analysis $2 \rightarrow \sigma_{\mathrm{cp}}=2,0 \mathrm{MPa}$ (3 tendons/meter) Results presented in Table 4.

\subsubsection{Designing from $\mathrm{ACl}$ 318-11}

Analysis $1 \rightarrow \sigma_{\mathrm{cp}}=1,33 \mathrm{MPa}$ (2 tendons/meter)

Results presented in Table 5.

Analysis $2 \rightarrow \sigma_{\mathrm{cp}}=2,0 \mathrm{MPa}$ (3 tendons/meter)

Results presented in Table 6 .

\section{Table 1}

Area of shear reinforcement $\left(\mathrm{cm}^{2}\right)$ by NBR 61 18:2007 (analysis 1)

\begin{tabular}{|c|c|c|c|c|c|c|c|c|c|c|c|}
\hline & & & $400 \mathrm{kN}$ & $500 \mathrm{kN}$ & $600 \mathrm{kN}$ & $700 \mathrm{kN}$ & $800 \mathrm{kN}$ & $900 \mathrm{kN}$ & $1000 \mathrm{kN}$ & $1100 \mathrm{kN}$ & $1200 \mathrm{kN}$ \\
\hline \multirow{6}{*}{$\begin{array}{c}\text { Column } \\
30 \times 30\end{array}$} & \multirow{2}{*}{ C 30} & $\rho=0,5 \%$ & 0,0 & 2,8 & 4,4 & 6,0 & 7,6 & 9,2 & 10,8 & 12,4 & - \\
\hline & & $\rho=1,5 \%$ & 0,0 & 0,0 & 2,6 & 4,2 & 5,8 & 7,4 & 9,0 & 10,6 & - \\
\hline & \multirow{2}{*}{ C 35} & $\rho=0,5 \%$ & 0,0 & 2,6 & 4,2 & 5,8 & 7,4 & 9,0 & 10,6 & 12,2 & 13,8 \\
\hline & & $\rho=1,5 \%$ & 0,0 & 0,0 & 2,3 & 3,9 & 5,5 & 7,1 & 8,7 & 10,3 & 11,9 \\
\hline & \multirow{2}{*}{ C 40} & $\rho=0,5 \%$ & 0,0 & 2,4 & 4,0 & 5,6 & 7,2 & 8,8 & 10,4 & 12,0 & 13,6 \\
\hline & & $\rho=1,5 \%$ & 0,0 & 0,0 & 2,1 & 3,7 & 5,3 & 6,9 & 8,5 & 10,1 & 11,7 \\
\hline \multirow{6}{*}{$\begin{array}{c}\text { Column } \\
40 \times 40\end{array}$} & \multirow{2}{*}{ C 30} & $\rho=0,5 \%$ & 0,0 & 2,3 & 3,9 & 5,5 & 7,1 & 8,7 & 10,3 & 11,9 & 13,5 \\
\hline & & $\rho=1,5 \%$ & 0,0 & 0,0 & 0,0 & 3,5 & 5,1 & 6,7 & 8,3 & 9,9 & 11,5 \\
\hline & \multirow{2}{*}{ C 35} & $\rho=0,5 \%$ & 0,0 & 2,0 & 3,6 & 5,2 & 6,8 & 8,4 & 10,0 & 11,6 & 13,2 \\
\hline & & $\rho=1,5 \%$ & 0,0 & 0,0 & 0,0 & 3,2 & 4,8 & 6,4 & 8,0 & 9,6 & 11,2 \\
\hline & \multirow{2}{*}{ C 40} & $\rho=0,5 \%$ & 0,0 & 1,8 & 3,4 & 5,0 & 6,6 & 8,2 & 9,8 & 11,4 & 13,0 \\
\hline & & $\rho=1,5 \%$ & 0,0 & 0,0 & 0,0 & 2,9 & 4,5 & 6,1 & 7,7 & 9,3 & 10,9 \\
\hline \multirow{6}{*}{$\begin{array}{l}\text { Column } \\
50 \times 50\end{array}$} & \multirow{2}{*}{ C 30} & $\rho=0,5 \%$ & 0,0 & 1,8 & 3,4 & 5,0 & 6,6 & 8,2 & 9,8 & 11,4 & 13,0 \\
\hline & & $\rho=1,5 \%$ & 0,0 & 0,0 & 0,0 & 2,8 & 4,4 & 6,0 & 7,6 & 9,2 & 10,8 \\
\hline & \multirow{2}{*}{ C 35} & $\rho=0,5 \%$ & 0,0 & 0,0 & 3,1 & 4,7 & 6,3 & 7,9 & 9,5 & 11,1 & 12,7 \\
\hline & & $\rho=1,5 \%$ & 0,0 & 0,0 & 0,0 & 2,4 & 4,0 & 5,6 & 7,2 & 8,8 & 10,4 \\
\hline & \multirow{2}{*}{ C 40} & $\rho=0,5 \%$ & 0,0 & 0,0 & 2,9 & 4,5 & 6,1 & 7,7 & 9,3 & 10,9 & 12,5 \\
\hline & & $\rho=1,5 \%$ & 0,0 & 0,0 & 0,0 & 0,0 & 3,6 & 5,2 & 6,8 & 8,4 & 10,0 \\
\hline
\end{tabular}


Table 2

Area of shear reinforcement ( $\mathrm{cm}^{2}$ ) by NBR 611 18:2007 (analysis 2)

\begin{tabular}{|c|c|c|c|c|c|c|c|c|c|c|c|}
\hline & & & $400 \mathrm{kN}$ & $500 \mathrm{kN}$ & $600 \mathrm{kN}$ & $700 \mathrm{kN}$ & $800 \mathrm{kN}$ & $900 \mathrm{kN}$ & $1000 \mathrm{kN}$ & $1100 \mathrm{kN}$ & $1200 \mathrm{kN}$ \\
\hline \multirow{6}{*}{$\begin{array}{l}\text { Column } \\
30 \times 30\end{array}$} & \multirow{2}{*}{ C 30} & $\rho=0,5 \%$ & 0,0 & 2,3 & 3,9 & 5,5 & 7,1 & 8,7 & 10,3 & 11,9 & - \\
\hline & & $\rho=1,5 \%$ & 0,0 & 0,0 & 2,1 & 3,7 & 5,3 & 6,9 & 8,5 & 10,1 & - \\
\hline & \multirow{2}{*}{ C 35} & $\rho=0,5 \%$ & 0,0 & 2,1 & 3,7 & 5,3 & 6,9 & 8,5 & 10,1 & 11,7 & 13,3 \\
\hline & & $\rho=1,5 \%$ & 0,0 & 0,0 & 0,0 & 3,4 & 5,0 & 6,6 & 8,2 & 9,8 & 11,4 \\
\hline & \multirow{2}{*}{ C 40} & $\rho=0,5 \%$ & 0,0 & 1,9 & 3,5 & 5,1 & 6,7 & 8,3 & 9,9 & 11,5 & 13,1 \\
\hline & & $\rho=1,5 \%$ & 0,0 & 0,0 & 0,0 & 3,2 & 4,8 & 6,4 & 8,0 & 9,6 & 11,2 \\
\hline \multirow{6}{*}{$\begin{array}{l}\text { Column } \\
40 \times 40\end{array}$} & \multirow{2}{*}{ C 30} & $\rho=0,5 \%$ & 0,0 & 1,8 & 3,4 & 5,0 & 6,6 & 8,2 & 9,8 & 11,4 & 13,0 \\
\hline & & $\rho=1,5 \%$ & 0,0 & 0,0 & 0,0 & 3,0 & 4,6 & 6,2 & 7,8 & 9,4 & 11,0 \\
\hline & \multirow{2}{*}{ C 35} & $\rho=0,5 \%$ & 0,0 & 1,5 & 3,1 & 4,7 & 6,3 & 7,9 & 9,5 & 11,1 & 12,7 \\
\hline & & $\rho=1,5 \%$ & 0,0 & 0,0 & 0,0 & 2,7 & 4,3 & 5,9 & 7,5 & 9,1 & 10,7 \\
\hline & \multirow{2}{*}{ C 40} & $\rho=0,5 \%$ & 0,0 & 0,0 & 2,9 & 4,5 & 6,1 & 7,7 & 9,3 & 10,9 & 12,5 \\
\hline & & $\rho=1,5 \%$ & 0,0 & 0,0 & 0,0 & 2,3 & 3,9 & 5,5 & 7,1 & 8,7 & 10,3 \\
\hline \multirow{6}{*}{$\begin{array}{l}\text { Column } \\
50 \times 50\end{array}$} & \multirow{2}{*}{ C 30} & $\rho=0,5 \%$ & 0,0 & 0,0 & 2,8 & 4,4 & 6,0 & 7,6 & 9,2 & 10,8 & 12,4 \\
\hline & & $\rho=1,5 \%$ & 0,0 & 0,0 & 0,0 & 2,3 & 3,9 & 5,5 & 7,1 & 8,7 & 10,3 \\
\hline & \multirow{2}{*}{ C 35} & $\rho=0,5 \%$ & 0,0 & 0,0 & 2,6 & 4,2 & 5,8 & 7,4 & 9,0 & 10,6 & 12,2 \\
\hline & & $\rho=1,5 \%$ & 0,0 & 0,0 & 0,0 & 0,0 & 3,5 & 5,1 & 6,7 & 8,3 & 9,9 \\
\hline & \multirow{2}{*}{ C 40} & $\rho=0,5 \%$ & 0,0 & 0,0 & 2,3 & 3,9 & 5,5 & 7,1 & 8,7 & 10,3 & 11,9 \\
\hline & & $\rho=1,5 \%$ & 0,0 & 0,0 & 0,0 & 0,0 & 3,1 & 4,7 & 6,3 & 7,9 & 9,5 \\
\hline
\end{tabular}

\section{Table 3}

Area of shear reinforcement ( $\mathrm{cm}^{2}$ ) by NBR 61 18:2014 (analysis 1)

\begin{tabular}{|c|c|c|c|c|c|c|c|c|c|c|c|}
\hline & & & $400 \mathrm{kN}$ & $500 \mathrm{kN}$ & $600 \mathrm{kN}$ & $700 \mathrm{kN}$ & $800 \mathrm{kN}$ & $900 \mathrm{kN}$ & $1000 \mathrm{kN}$ & $1100 \mathrm{kN}$ & $1200 \mathrm{kN}$ \\
\hline \multirow{6}{*}{$\begin{array}{c}\text { Column } \\
30 \times 30\end{array}$} & \multirow{2}{*}{ C 30} & $\rho=0,5 \%$ & 0,0 & 1,8 & 3,4 & 5,0 & 6,6 & 8,2 & 9,8 & 11,4 & - \\
\hline & & $\rho=1,5 \%$ & 0,0 & 0,0 & 0,0 & 3,2 & 4,8 & 6,4 & 8,0 & 9,6 & - \\
\hline & \multirow{2}{*}{ C 35} & $\rho=0,5 \%$ & 0,0 & 1,6 & 3,2 & 4,8 & 6,4 & 8,0 & 9,6 & 11,2 & 12,8 \\
\hline & & $\rho=1,5 \%$ & 0,0 & 0,0 & 0,0 & 2,9 & 4,5 & 6,1 & 7,7 & 9,3 & 10,9 \\
\hline & \multirow{2}{*}{ C 40} & $\rho=0,5 \%$ & 0,0 & 1,4 & 3,0 & 4,6 & 6,2 & 7,8 & 9,4 & 11,0 & 12,6 \\
\hline & & $\rho=1,5 \%$ & 0,0 & 0,0 & 0,0 & 2,7 & 4,3 & 5,9 & 7,5 & 9,1 & 10,7 \\
\hline \multirow{6}{*}{$\begin{array}{c}\text { Column } \\
40 \times 40\end{array}$} & \multirow{2}{*}{ C 30} & $\rho=0,5 \%$ & 0,0 & 0,0 & 2,7 & 4,3 & 5,9 & 7,5 & 9,1 & 10,7 & 12,3 \\
\hline & & $\rho=1,5 \%$ & 0,0 & 0,0 & 0,0 & 2,4 & 4,0 & 5,6 & 7,2 & 8,8 & 10,4 \\
\hline & \multirow{2}{*}{ C 35} & $\rho=0,5 \%$ & 0,0 & 0,0 & 2,5 & 4,1 & 5,7 & 7,3 & 8,9 & 10,5 & 12,1 \\
\hline & & $\rho=1,5 \%$ & 0,0 & 0,0 & 0,0 & 0,0 & 3,6 & 5,2 & 6,8 & 8,4 & 10,0 \\
\hline & \multirow{2}{*}{ C 40} & $\rho=0,5 \%$ & 0,0 & 0,0 & 2,3 & 3,9 & 5,5 & 7,1 & 8,7 & 10,3 & 11,9 \\
\hline & & $\rho=1,5 \%$ & 0,0 & 0,0 & 0,0 & 0,0 & 3,3 & 4,9 & 6,5 & 8,1 & 9,7 \\
\hline \multirow{6}{*}{$\begin{array}{c}\text { Column } \\
50 \times 50\end{array}$} & \multirow{2}{*}{ C 30} & $\rho=0,5 \%$ & 0,0 & 0,0 & 2,1 & 3,7 & 5,3 & 6,9 & 8,5 & 10,1 & 11,7 \\
\hline & & $\rho=1,5 \%$ & 0,0 & 0,0 & 0,0 & 0,0 & 3,1 & 4,7 & 6,3 & 7,9 & 9,5 \\
\hline & \multirow{2}{*}{ C 35} & $\rho=0,5 \%$ & 0,0 & 0,0 & 1,8 & 3,4 & 5,0 & 6,6 & 8,2 & 9,8 & 11,4 \\
\hline & & $\rho=1,5 \%$ & 0,0 & 0,0 & 0,0 & 0,0 & 2,7 & 4,3 & 5,9 & 7,5 & 9,1 \\
\hline & \multirow{2}{*}{ C 40} & $\rho=0,5 \%$ & 0,0 & 0,0 & 0,0 & 3,2 & 4,8 & 6,4 & 8,0 & 9,6 & 11,2 \\
\hline & & $\rho=1,5 \%$ & 0,0 & 0,0 & 0,0 & 0,0 & 0,0 & 4,0 & 5,6 & 7,2 & 8,8 \\
\hline
\end{tabular}




\subsubsection{Designing from Eurocode 2:2004}

Analysis $1 \rightarrow \sigma_{\mathrm{cp}}=1,33 \mathrm{MPa}$ (2 tendons/meter)

Results presented in Table 7.

Analysis $2 \rightarrow \sigma_{c p}=2,0 \mathrm{MPa}$ (3 tendons/meter)

Results presented in Table 8 .

\subsection{Comparative analysis}

Tables 9 to 20 show the percentage reductions in the calculated reinforcement when comparing the codes studied. There are cells without values because null results are not being compared or those that exceed the design limits.

\section{a) Comparison 1}

The Tables 9 and 10 show the percentage reductions of punching shear reinforcement obtained when the design using NBR 6118:2007 is replaced by using NBR 6118:2014.

When analyzed the relations between the two codes, it is seen that there is a significant difference in the level of conservatism among them being the current code is more economical, especially at lower loading levels.

The gains generated by NBR 6118: 2014 increases in the case of columns with larger cross sections, considering the same loading. Moreover, the efficiency of this standard also improves when the prestressing level increases.
The increase in flexural reinforcement ratio generates a reduction of approximately $5 \%$ to $10 \%$ on the calculated shear reinforcement.

On the other hand, as the load increases, the savings generated by NBR 6118:2014 decreases compared to the results generated by NBR 6118:2007, but still resulting in smaller shear reinforcement area.

\section{b) Comparison 2}

The Tables 11 and 12 show the percentage reductions of punching shear reinforcement obtained when the design using NBR 6118:2007 is replaced by using EC2:2004.

When EC2 is compared to NBR $6118: 2007$, there is a big difference in results obtained by the two codes, and it is noticed that the European code is more economical.

The European code is more advantageous with increasing section of the columns, keeping the load constant. Furthermore, increasing the compressive stress in the slab plane, the Eurocode efficiency increases even more, compared with the Brazilian standard.

It is important to note that the minimum reduction obtained by EC2 has been around $30 \%$ and the maximum was $95 \%$, for the values that are comparable, showing that this code has formulations that generate boldest results.

In general, increasing the shear force the difference between the standards is reduced. For the highest loads, raise the flexural reinforcement ratio does not generate a significant reduction in

\section{Table 4}

Area of shear reinforcement ( $\mathrm{cm}^{2}$ ) by NBR 611 18:2014 (analysis 2)

\begin{tabular}{|c|c|c|c|c|c|c|c|c|c|c|c|}
\hline & & & $400 \mathrm{kN}$ & $500 \mathrm{kN}$ & $600 \mathrm{kN}$ & $700 \mathrm{kN}$ & $800 \mathrm{kN}$ & $900 \mathrm{kN}$ & $1000 \mathrm{kN}$ & $1100 \mathrm{kN}$ & $1200 \mathrm{kN}$ \\
\hline \multirow{6}{*}{$\begin{array}{l}\text { Column } \\
30 \times 30\end{array}$} & \multirow{2}{*}{ C 30} & $\rho=0,5 \%$ & 0,0 & 0,0 & 2,4 & 4,0 & 5,6 & 7,2 & 8,8 & 10,4 & - \\
\hline & & $\rho=1,5 \%$ & 0,0 & 0,0 & 0,0 & 2,3 & 3,9 & 5,5 & 7,1 & 8,7 & - \\
\hline & \multirow{2}{*}{ C 35} & $\rho=0,5 \%$ & 0,0 & 0,0 & 2,2 & 3,8 & 5,4 & 7,0 & 8,6 & 10,2 & 11,8 \\
\hline & & $\rho=1,5 \%$ & 0,0 & 0,0 & 0,0 & 2,0 & 3,6 & 5,2 & 6,8 & 8,4 & 10,0 \\
\hline & \multirow{2}{*}{ C 40} & $\rho=0,5 \%$ & 0,0 & 0,0 & 2,0 & 3,6 & 5,2 & 6,8 & 8,4 & 10,0 & 11,6 \\
\hline & & $\rho=1,5 \%$ & 0,0 & 0,0 & 0,0 & 0,0 & 3,3 & 4,9 & 6,5 & 8,1 & 9,7 \\
\hline \multirow{6}{*}{$\begin{array}{l}\text { Column } \\
40 \times 40\end{array}$} & \multirow{2}{*}{ C 30} & $\rho=0,5 \%$ & 0,0 & 0,0 & 1,7 & 3,3 & 4,9 & 6,5 & 8,1 & 9,7 & 11,3 \\
\hline & & $\rho=1,5 \%$ & 0,0 & 0,0 & 0,0 & 0,0 & 2,9 & 4,5 & 6,1 & 7,7 & 9,3 \\
\hline & \multirow{2}{*}{ C 35} & $\rho=0,5 \%$ & 0,0 & 0,0 & 1,5 & 3,1 & 4,7 & 6,3 & 7,9 & 9,5 & 11,1 \\
\hline & & $\rho=1,5 \%$ & 0,0 & 0,0 & 0,0 & 0,0 & 2,6 & 4,2 & 5,8 & 7,4 & 9,0 \\
\hline & \multirow{2}{*}{ C 40} & $\rho=0,5 \%$ & 0,0 & 0,0 & 0,0 & 2,8 & 4,4 & 6,0 & 7,6 & 9,2 & 10,8 \\
\hline & & $\rho=1,5 \%$ & 0,0 & 0,0 & 0,0 & 0,0 & 2,3 & 3,9 & 5,5 & 7,1 & 8,7 \\
\hline \multirow{6}{*}{$\begin{array}{l}\text { Column } \\
50 \times 50\end{array}$} & \multirow{2}{*}{ C 30} & $\rho=0,5 \%$ & 0,0 & 0,0 & 0,0 & 2,6 & 4,2 & 5,8 & 7,4 & 9,0 & 10,6 \\
\hline & & $\rho=1,5 \%$ & 0,0 & 0,0 & 0,0 & 0,0 & 0,0 & 3,6 & 5,2 & 6,8 & 8,4 \\
\hline & \multirow{2}{*}{ C 35} & $\rho=0,5 \%$ & 0,0 & 0,0 & 0,0 & 2,3 & 3,9 & 5,5 & 7,1 & 8,7 & 10,3 \\
\hline & & $\rho=1,5 \%$ & 0,0 & 0,0 & 0,0 & 0,0 & 0,0 & 3,2 & 4,8 & 6,4 & 8,0 \\
\hline & \multirow{2}{*}{ C 40} & $\rho=0,5 \%$ & 0,0 & 0,0 & 0,0 & 2,1 & 3,7 & 5,3 & 6,9 & 8,5 & 10,1 \\
\hline & & $\rho=1,5 \%$ & 0,0 & 0,0 & 0,0 & 0,0 & 0,0 & 2,9 & 4,5 & 6,1 & 7,7 \\
\hline
\end{tabular}




\section{Table 5}

Area of shear reinforcement $\left(\mathrm{cm}^{2}\right)$ by ACl 318-11 (analysis 1)

\begin{tabular}{|c|c|c|c|c|c|c|c|c|c|c|c|}
\hline & & & $400 \mathrm{kN}$ & $500 \mathrm{kN}$ & $600 \mathrm{kN}$ & $700 \mathrm{kN}$ & $800 \mathrm{kN}$ & $900 \mathrm{kN}$ & $1000 \mathrm{kN}$ & $1100 \mathrm{kN}$ & $1200 \mathrm{kN}$ \\
\hline \multirow{6}{*}{$\begin{array}{c}\text { Column } \\
30 \times 30\end{array}$} & \multirow{2}{*}{ C 30} & $\rho=0,5 \%$ & \multirow{2}{*}{0,0} & \multirow{2}{*}{6,6} & \multirow{2}{*}{8,7} & \multirow{2}{*}{10,9} & \multirow{2}{*}{ - } & \multirow{2}{*}{ - } & \multirow{2}{*}{ - } & \multirow{2}{*}{ - } & \multirow{2}{*}{ - } \\
\hline & & $\rho=1,5 \%$ & & & & & & & & & \\
\hline & \multirow{2}{*}{ C 35} & $\rho=0,5 \%$ & \multirow{2}{*}{0,0} & \multirow{2}{*}{6,3} & \multirow{2}{*}{8,4} & \multirow{2}{*}{10,5} & \multirow{2}{*}{12,6} & \multirow{2}{*}{ - } & \multirow{2}{*}{-} & \multirow{2}{*}{ - } & \multirow{2}{*}{ - } \\
\hline & & $\rho=1,5 \%$ & & & & & & & & & \\
\hline & \multirow{2}{*}{ C 40} & $\rho=0,5 \%$ & \multirow{2}{*}{0,0} & \multirow{2}{*}{6,0} & \multirow{2}{*}{8,1} & \multirow{2}{*}{10,3} & \multirow{2}{*}{12,3} & \multirow{2}{*}{-} & \multirow{2}{*}{ - } & \multirow{2}{*}{-} & \multirow{2}{*}{ - } \\
\hline & & $\rho=1,5 \%$ & & & & & & & & & \\
\hline \multirow{6}{*}{$\begin{array}{c}\text { Column } \\
40 \times 40\end{array}$} & \multirow{2}{*}{ C 30} & $\rho=0,5 \%$ & \multirow{2}{*}{0,0} & م0 & 70 & 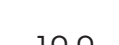 & 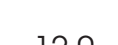 & 740 & & & \\
\hline & & $\rho=1,5 \%$ & & 0,0 & 1,0 & 10,0 & 12,0 & 14,2 & - & - & - \\
\hline & 1035 & $\rho=0,5 \%$ & مת & مि & 71 & $\Omega^{\prime}$ & 7 & 120 & & & \\
\hline & & $\rho=1,5 \%$ & 0,0 & 0,0 & 1,4 & 4,0 & 11,7 & 10,0 & - & - & - \\
\hline & 010 & $\rho=0,5 \%$ & مि & م0 & 71 & ? & 7 & 121 & 155 & & \\
\hline & $\subset-40$ & $\rho=1,5 \%$ & 0,0 & 0,0 & 7,1 & प, & & & & & \\
\hline & $0^{3}$ & $\rho=0,5 \%$ & مि & م0 & م0 & 1 & $1] 2$ & 133 & 151 & & \\
\hline & C & $\rho=1,5 \%$ & 0,0 & 0,0 & 0,0 & 3,1 & & & & & \\
\hline Column & $C_{35}$ & $\rho=0,5 \%$ & مि & مחم & مח & 86 & 107 & 128 & 310 & 171 & - \\
\hline $50 \times 50$ & C & $\rho=1,5 \%$ & 0,0 & 0,0 & 0,0 & 0,0 & 0,1 & 12,0 & 14,7 & 11,1 & - \\
\hline & 1010 & $\rho=0,5 \%$ & ת & ת & R & $8 ?$ & 103 & 121 & 34 & 166 & 188 \\
\hline & 1040 & $\rho=1,5 \%$ & 0,0 & 0,0 & 0,0 & 0,2 & (1) & 12,4 & 14,6 & 10,0 & 10,0 \\
\hline
\end{tabular}

\section{Table 6}

Area of shear reinforcement $\left(\mathrm{cm}^{2}\right)$ by ACl 318-11 (analysis 2)

\begin{tabular}{|c|c|c|c|c|c|c|c|c|c|c|c|}
\hline & & & $400 \mathrm{kN}$ & $500 \mathrm{kN}$ & $600 \mathrm{kN}$ & $700 \mathrm{kN}$ & $800 \mathrm{kN}$ & $900 \mathrm{kN}$ & $1000 \mathrm{kN}$ & $1100 \mathrm{kN}$ & $1200 \mathrm{kN}$ \\
\hline \multirow{6}{*}{$\begin{array}{c}\text { Column } \\
30 \times 30\end{array}$} & \multirow{2}{*}{ C 30} & $\rho=0,5 \%$ & \multirow{2}{*}{0,0} & \multirow{2}{*}{0,0} & \multirow{2}{*}{8,7} & \multirow{2}{*}{10,9} & \multirow{2}{*}{-} & \multirow{2}{*}{-} & \multirow{2}{*}{ - } & \multirow{2}{*}{ - } & \multirow{2}{*}{ - } \\
\hline & & $\rho=1,5 \%$ & & & & & & & & & \\
\hline & \multirow{2}{*}{ C 35} & $\rho=0,5 \%$ & \multirow{2}{*}{0,0} & \multirow{2}{*}{0,0} & \multirow{2}{*}{8,4} & \multirow{2}{*}{10,6} & \multirow{2}{*}{12,6} & \multirow{2}{*}{ - } & \multirow{2}{*}{ - } & \multirow{2}{*}{ - } & \multirow{2}{*}{ - } \\
\hline & & $\rho=1,5 \%$ & & & & & & & & & \\
\hline & \multirow{2}{*}{ C 40} & $\rho=0,5 \%$ & \multirow{2}{*}{0,0} & \multirow{2}{*}{0,0} & \multirow{2}{*}{8,1} & \multirow{2}{*}{10,3} & \multirow{2}{*}{12,3} & \multirow{2}{*}{ - } & \multirow{2}{*}{ - } & \multirow{2}{*}{ - } & \multirow{2}{*}{ - } \\
\hline & & $\rho=1,5 \%$ & & & & & & & & & \\
\hline \multirow{6}{*}{$\begin{array}{c}\text { Column } \\
40 \times 40\end{array}$} & \multirow{2}{*}{ C 30} & $\rho=0,5 \%$ & \multirow{2}{*}{0,0} & & & & & & & & \\
\hline & & $\rho=1,5 \%$ & & 0,0 & 0,0 & 10,0 & 12,0 & 14,2 & - & - & - \\
\hline & 005 & $\rho=0,5 \%$ & 80 & 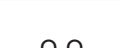 & 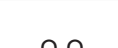 & 0 & 7 & 720 & & & \\
\hline & C & $\rho=1,5 \%$ & 0,0 & 0,0 & 0,0 & 4,0 & 11,1 & 10,0 & - & - & - \\
\hline & $\mathrm{C}_{4}$ & $\rho=0,5 \%$ & م० & م० & م० & 92 & 113 & 134 & 155 & - & - \\
\hline & & $\rho=1,5 \%$ & & & & & & & & & \\
\hline & 1000 & $\rho=0,5 \%$ & Pि० & 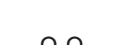 & 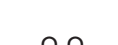 & 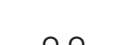 & 7 & 720 & 75 & & \\
\hline & Cos & $\rho=1,5 \%$ & 0,0 & 0,0 & 0,0 & 0,0 & 11,2 & 10,0 & 10,4 & - & - \\
\hline Column & $C_{35}$ & $\rho=0,5 \%$ & مी & م0 & م0 & תم & 7 & 120 & 140 & 17 & \\
\hline $50 \times 50$ & C & $\rho=1,5 \%$ & 0,0 & 0,0 & 0,0 & 0,0 & 0,1 & 12,0 & 14,7 & 11,1 & - \\
\hline & $c_{10}$ & $\rho=0,5 \%$ & مि & مि & مח & مि & 103 & 121 & 315 & 166 & 100 \\
\hline & $\subset-40$ & $\rho=1,5 \%$ & 0,0 & 0,0 & 0,0 & 0,0 & 10,0 & 12,4 & 14,0 & 10,0 & 10,0 \\
\hline
\end{tabular}




\section{Table 7}

Area of shear reinforcement ( $\left.\mathrm{cm}^{2}\right)$ by EC2:2004 (analysis 1)

\begin{tabular}{|c|c|c|c|c|c|c|c|c|c|c|c|}
\hline & & & $400 \mathrm{kN}$ & $500 \mathrm{kN}$ & $600 \mathrm{kN}$ & $700 \mathrm{kN}$ & $800 \mathrm{kN}$ & $900 \mathrm{kN}$ & $1000 \mathrm{kN}$ & $1100 \mathrm{kN}$ & $1200 \mathrm{kN}$ \\
\hline \multirow{6}{*}{$\begin{array}{c}\text { Column } \\
30 \times 30\end{array}$} & \multirow{2}{*}{ C 30} & $\rho=0,5 \%$ & 0,0 & 0,8 & 2,1 & 3,5 & 4,8 & 6,2 & 7,5 & - & - \\
\hline & & $\rho=1,5 \%$ & 0,0 & 0,0 & 0,0 & 2,2 & 3,5 & 4,8 & 6,2 & - & - \\
\hline & \multirow{2}{*}{ C 35} & $\rho=0,5 \%$ & 0,0 & 0,6 & 1,9 & 3,3 & 4,6 & 6,0 & 7,3 & 8,7 & - \\
\hline & & $\rho=1,5 \%$ & 0,0 & 0,0 & 0,0 & 1,9 & 3,3 & 4,6 & 5,9 & 7,3 & - \\
\hline & \multirow{2}{*}{ C 40} & $\rho=0,5 \%$ & 0,0 & 0,0 & 1,8 & 3,2 & 4,5 & 5,8 & 7,2 & 8,5 & 9,9 \\
\hline & & $\rho=1,5 \%$ & 0,0 & 0,0 & 0,0 & 1,7 & 3,1 & 4,4 & 5,8 & 7,1 & 8,4 \\
\hline \multirow{6}{*}{$\begin{array}{c}\text { Column } \\
40 \times 40\end{array}$} & \multirow{2}{*}{ C 30} & $\rho=0,5 \%$ & 0,0 & 0,0 & 1,5 & 2,9 & 4,2 & 5,6 & 6,9 & 8,3 & 9,6 \\
\hline & & $\rho=1,5 \%$ & 0,0 & 0,0 & 0,0 & 1,4 & 2,8 & 4,1 & 5,4 & 6,8 & 8,1 \\
\hline & \multirow{2}{*}{ C 35} & $\rho=0,5 \%$ & 0,0 & 0,0 & 1,4 & 2,7 & 4,0 & 5,4 & 6,7 & 8,1 & 9,4 \\
\hline & & $\rho=1,5 \%$ & 0,0 & 0,0 & 0,0 & 1,2 & 2,5 & 3,8 & 5,2 & 6,5 & 7,9 \\
\hline & \multirow{2}{*}{ C 40} & $\rho=0,5 \%$ & 0,0 & 0,0 & 1,2 & 2,6 & 3,9 & 5,2 & 6,6 & 7,9 & 9,3 \\
\hline & & $\rho=1,5 \%$ & 0,0 & 0,0 & 0,0 & 0,0 & 2,2 & 3,6 & 4,9 & 6,3 & 7,6 \\
\hline \multirow{6}{*}{$\begin{array}{l}\text { Column } \\
50 \times 50\end{array}$} & \multirow{2}{*}{ C 30} & $\rho=0,5 \%$ & 0,0 & 0,0 & 1,0 & 2,3 & 3,7 & 5,0 & 6,3 & 7,7 & 9,0 \\
\hline & & $\rho=1,5 \%$ & 0,0 & 0,0 & 0,0 & 0,0 & 2,0 & 3,4 & 4,7 & 6,0 & 7,4 \\
\hline & \multirow{2}{*}{ C 35} & $\rho=0,5 \%$ & 0,0 & 0,0 & 0,8 & 2,1 & 3,5 & 4,8 & 6,1 & 7,5 & 8,8 \\
\hline & & $\rho=1,5 \%$ & 0,0 & 0,0 & 0,0 & 0,0 & 1,7 & 3,1 & 4,4 & 5,8 & 7,1 \\
\hline & \multirow{2}{*}{ C 40} & $\rho=0,5 \%$ & 0,0 & 0,0 & 0,0 & 1,9 & 3,3 & 4,6 & 6,0 & 7,3 & 8,6 \\
\hline & & $\rho=1,5 \%$ & 0,0 & 0,0 & 0,0 & 0,0 & 1,4 & 2,8 & 4,1 & 5,5 & 6,8 \\
\hline
\end{tabular}

\section{Table 8}

Area of shear reinforcement $\left(\mathrm{cm}^{2}\right)$ by EC2:2004 (analysis 2)

\begin{tabular}{|c|c|c|c|c|c|c|c|c|c|c|c|}
\hline & & & $400 \mathrm{kN}$ & $500 \mathrm{kN}$ & $600 \mathrm{kN}$ & $700 \mathrm{kN}$ & $800 \mathrm{kN}$ & $900 \mathrm{kN}$ & $1000 \mathrm{kN}$ & $1100 \mathrm{kN}$ & $1200 \mathrm{kN}$ \\
\hline \multirow{6}{*}{$\begin{array}{l}\text { Column } \\
30 \times 30\end{array}$} & \multirow{2}{*}{ C 30} & $\rho=0,5 \%$ & 0,0 & 0,0 & 0,8 & 2,2 & 3,5 & 4,8 & 6,2 & - & - \\
\hline & & $\rho=1,5 \%$ & 0,0 & 0,0 & 0,0 & 0,8 & 2,2 & 3,6 & 4,9 & - & - \\
\hline & \multirow{2}{*}{ C 35} & $\rho=0,5 \%$ & 0,0 & 0,0 & 0,7 & 2,0 & 3,3 & 4,7 & 6,0 & 7,4 & - \\
\hline & & $\rho=1,5 \%$ & 0,0 & 0,0 & 0,0 & 0,6 & 2,0 & 3,3 & 4,7 & 6,0 & - \\
\hline & \multirow{2}{*}{ C 40} & $\rho=0,5 \%$ & 0,0 & 0,0 & 0,5 & 1,9 & 3,2 & 4,5 & 5,9 & 7,2 & 8,6 \\
\hline & & $\rho=1,5 \%$ & 0,0 & 0,0 & 0,0 & 0,0 & 1,7 & 3,1 & 4,5 & 5,8 & 7,1 \\
\hline \multirow{6}{*}{$\begin{array}{l}\text { Column } \\
40 \times 40\end{array}$} & \multirow{2}{*}{ C 30} & $\rho=0,5 \%$ & 0,0 & 0,0 & 0,2 & 1,5 & 2,9 & 4,2 & 5,5 & 6,9 & 8,2 \\
\hline & & $\rho=1,5 \%$ & 0,0 & 0,0 & 0,0 & 0,0 & 1,4 & 2,7 & 4,1 & 5,4 & 6,8 \\
\hline & \multirow{2}{*}{ C 35} & $\rho=0,5 \%$ & 0,0 & 0,0 & 0,0 & 1,3 & 2,7 & 4,0 & 5,4 & 6,7 & 8,0 \\
\hline & & $\rho=1,5 \%$ & 0,0 & 0,0 & 0,0 & 0,0 & 1,1 & 2,4 & 3,8 & 5,1 & 6,5 \\
\hline & \multirow{2}{*}{ C 40} & $\rho=0,5 \%$ & 0,0 & 0,0 & 0,0 & 1,1 & 2,5 & 3,8 & 5,2 & 6,5 & 7,9 \\
\hline & & $\rho=1,5 \%$ & 0,0 & 0,0 & 0,0 & 0,0 & 0,9 & 2,2 & 3,6 & 4,9 & 6,3 \\
\hline \multirow{6}{*}{$\begin{array}{l}\text { Column } \\
50 \times 50\end{array}$} & \multirow{2}{*}{ C 30} & $\rho=0,5 \%$ & 0,0 & 0,0 & 0,0 & 0,9 & 2,2 & 3,5 & 4,9 & 6,2 & 7,6 \\
\hline & & $\rho=1,5 \%$ & 0,0 & 0,0 & 0,0 & 0,0 & 0,0 & 1,9 & 3,2 & 4,5 & 5,9 \\
\hline & \multirow{2}{*}{ C 35} & $\rho=0,5 \%$ & 0,0 & 0,0 & 0,0 & 0,7 & 2,0 & 3,3 & 4,7 & 6,0 & 7,4 \\
\hline & & $\rho=1,5 \%$ & 0,0 & 0,0 & 0,0 & 0,0 & 0,0 & 1,6 & 2,9 & 4,3 & 5,6 \\
\hline & \multirow{2}{*}{ C 40} & $\rho=0,5 \%$ & 0,0 & 0,0 & 0,0 & 0,5 & 1,8 & 3,1 & 4,5 & 5,8 & 7,2 \\
\hline & & $\rho=1,5 \%$ & 0,0 & 0,0 & 0,0 & 0,0 & 0,0 & 1,3 & 2,7 & 4,0 & 5,4 \\
\hline
\end{tabular}


calculated reinforcement shear, however a reduction of $10 \%$ to $20 \%$ is achieved when the load decreases.

\section{c) Comparison 3}

The Tables 13 and 14 show the percentage reductions of punching shear reinforcement obtained when the design using NBR 6118:2014 is replaced by using EC2:2004.

The European code provides lower values of shear reinforcement to all situations compared, noting that, for smaller loads, the difference between the standards becomes even more significant. For compressive strain $\left(\sigma_{c p}\right)$ equal to $1,33 \mathrm{MPa}$, it is observed that the difference between them tends to stabilize at $20 \%$ by increasing the load. Considering the slabs with $\sigma_{\mathrm{cp}}$ equal to $2,0 \mathrm{MPa}$, the values calculated by EC2 tend to be $30 \%$ lower than those calculated by the Brazilian standard, increasing the load.

For slabs with smaller prestressing force, raising the flexural reinforcement ratio does not generate a significant reduction to the calculated punching shear reinforcement. However, for slabs with $\sigma_{c p}$ equal to $2,0 \mathrm{MPa}$, increase the flexural reinforcement causes a expressive reduction in shear reinforcement for loads up to $800 \mathrm{kN}$.

\section{d) Comparison 4}

The Tables 15 and 16 show the percentage reductions of punching shear reinforcement obtained when the design using $\mathrm{ACl} 318-11$ is replaced by using EC2:2004.

Analyzing the four standards compared in this study, it can be said that the greater difference is found between in the designs EC2 and $\mathrm{ACl} 318$. The minimum savings generated by the European code was $54 \%$ compared to the American standard. For smaller loads, the punching shear reinforcement shear can be reduced by more than $90 \%$.

It also observes that for larger sections of columns, keeping constant loading, the economy generated by EC2 tends to increase.

In the theoretical examples provided, elevation of prestressing increases the savings generated using Eurocode in about $10 \%$. Also, raising the flexural reinforcement ratio generates a reduction about $10 \%$ to $15 \%$ for the calculated punching shear reinforcement.

\section{e) Comparison 5}

The Tables 17 and 18 show the percentage reductions of punching shear reinforcement obtained when the design using ACI318-11 is replaced by using NBR 6118:2007.

The results of the comparison between the NBR 6118:2007 and $\mathrm{ACl} 318$ show that Brazilian standard is more efficient than the American, with reductions greater than $40 \%$ on the calculated punching shear reinforcement. It is achieved greater reduction in the reinforcement for smaller loads, as well as the other comparisons.

Even though the variation in $\sigma_{\mathrm{cp}}$ does not significantly change the relationship between the two codes, raising the flexural reinforcement generates a reduction of $15 \%$ to $20 \%$ for shear reinforcement when calculated by the NBR $6118: 2007$. It is also noted that the savings generated by the Brazilian standard grows with increasing section of the pillars and constant loads.

\section{f) Comparison 6}

The Tables 19 and 20 show the percentage reductions of punching shear reinforcement obtained when the design using ACl318-11 is replaced by using NBR 6118:2014.

It is observed that the difference between the two standards is about $70 \%$ for smaller loads and $50 \%$ for higher loads.

The economy generated by NBR 6118:2014 grows by increasing the section of the pillars and constant loads. There is also an approximate $10 \%$ increase in the savings obtained by NBR 6118:2014 when the prestressing is elevated and this fact can be observed for all values compared in the results tables.

The increase in flexural reinforcement generates a reduction rate of $15 \%$ to $20 \%$ on the calculated shear reinforcement.

\subsection{Maximum loads without punching shear reinforcement}

Table 21 shows the maximum factored shear force for each case, and these cases are numbered at the last column of the table.

Observing the maximum loads without punching shear reinforcement, it can be noticed that $\mathrm{ACl}$ 318-11 does not take into account the variation of the flexural reinforcement ratio $(\rho)$, so for the 18 cases analyzed, the results vary only with the compression strength of concrete and with the section of supporting columns. Considering $\rho$ equal to $1.5 \%$, the results of $\mathrm{ACl}$ and NBR 6118:2007 are always close to each other and also the most conservative.

In general, for the odd cases (low flexural reinforcement ratios), NBR 6118: 2007 presents the most conservative results and $\mathrm{ACl}$ leads to results bolder than other standards, particularly for larger sections columns. For low reinforcement ratios, only in the cases 1, 3 and 5 EC2:2004 reached load values higher than ACI 318-11. Eurocode and NBR 6118:2014 also generate very similar values in all cases compared and always achieve the highest maximum shear force when $\rho$ is equal to $1.5 \%$.

Increasing prestressing had less influence to NBR 6118:2007, resulting an increase in the maximum load resistant approximately of $5 \%$. However, the other three design codes had an increase of $10 \%$ to $15 \%$ in puncture resistance slab.

\section{Final considerations}

\subsection{Punching shear reinforcement calculated by theoretical examples}

Initially, it may be affirmed that for all cases, as the load on the slab rises, the necessary punching shear reinforcement have also an increase.

There is a reduction of the shear reinforcement calculated increasing the section of the columns and concrete strength.

For $\mathrm{ACl} 318$, increasing the reinforcement ratio does not influence the shear resistance, but for NBR 6118:2007, NBR 6118:2014 and $E C 2$, there is a considerable decrease in the required punching shear reinforcement. 


\section{Table 9}

Percentual reduction by Comparison 1, considering $\sigma_{\mathrm{cp}}=1,33 \mathrm{MPa}$

\begin{tabular}{|c|c|c|c|c|c|c|c|c|c|c|c|}
\hline & & & $400 \mathrm{kN}$ & $500 \mathrm{kN}$ & $600 \mathrm{kN}$ & $700 \mathrm{kN}$ & $800 \mathrm{kN}$ & $900 \mathrm{kN}$ & $1000 \mathrm{kN}$ & $1100 \mathrm{kN}$ & $1200 \mathrm{kN}$ \\
\hline \multirow{6}{*}{$\begin{array}{c}\text { Column } \\
30 \times 30\end{array}$} & \multirow{2}{*}{ C 30} & $\rho=0,5 \%$ & - & 35,7 & 22,7 & 16,7 & 13,2 & 10,9 & 9,3 & 8,1 & - \\
\hline & & $\rho=1,5 \%$ & - & - & - & 23,8 & 17,2 & 13,5 & 11,1 & 9,4 & - \\
\hline & \multirow{2}{*}{ C 35} & $\rho=0,5 \%$ & - & 38,5 & 23,8 & 17,2 & 13,5 & 11,1 & 9,4 & 8,2 & 7,2 \\
\hline & & $\rho=1,5 \%$ & - & - & - & 25,6 & 18,2 & 14,1 & 11,5 & 9,7 & 8,4 \\
\hline & \multirow{2}{*}{ C 40} & $\rho=0,5 \%$ & - & 41,7 & 25,0 & 17,9 & 13,9 & 11,4 & 9,6 & 8,3 & 7,4 \\
\hline & & $\rho=1,5 \%$ & - & - & - & 27,0 & 18,9 & 14,5 & 11,8 & 9,9 & 8,5 \\
\hline \multirow{6}{*}{$\begin{array}{c}\text { Column } \\
40 \times 40\end{array}$} & \multirow{2}{*}{ C 30} & $\rho=0,5 \%$ & - & - & 30,8 & 21,8 & 16,9 & 13,8 & 11,7 & 10,1 & 8,9 \\
\hline & & $\rho=1,5 \%$ & - & - & - & 31,4 & 21,6 & 16,4 & 13,3 & 11,1 & 9,6 \\
\hline & \multirow{2}{*}{ C 35} & $\rho=0,5 \%$ & - & - & 30,6 & 21,2 & 16,2 & 13,1 & 11,0 & 9,5 & 8,3 \\
\hline & & $\rho=1,5 \%$ & - & - & - & - & 25,0 & 18,8 & 15,0 & 12,5 & 10,7 \\
\hline & \multirow{2}{*}{ C 40} & $\rho=0,5 \%$ & - & - & 32,4 & 22,0 & 16,7 & 13,4 & 11,2 & 9,6 & 8,5 \\
\hline & & $\rho=1,5 \%$ & - & - & - & - & 26,7 & 19,7 & 15,6 & 12,9 & 11,0 \\
\hline \multirow{6}{*}{$\begin{array}{c}\text { Column } \\
50 \times 50\end{array}$} & \multirow{2}{*}{ C 30} & $\rho=0,5 \%$ & - & - & 38,2 & 26,0 & 19,7 & 15,9 & 13,3 & 11,4 & 10,0 \\
\hline & & $\rho=1,5 \%$ & - & - & - & - & 29,5 & 21,7 & 17,1 & 14,1 & 12,0 \\
\hline & \multirow{2}{*}{ C 35} & $\rho=0,5 \%$ & - & - & 41,9 & 27,7 & 20,6 & 16,5 & 13,7 & 11,7 & 10,2 \\
\hline & & $\rho=1,5 \%$ & - & - & - & & 32,5 & 23,2 & 18,1 & 14,8 & 12,5 \\
\hline & \multirow{2}{*}{ C 40} & $\rho=0,5 \%$ & - & - & - & 28,9 & 21,3 & 16,9 & 14,0 & 11,9 & 10,4 \\
\hline & & $\rho=1,5 \%$ & - & - & - & - & - & 23,1 & 17,6 & 14,3 & 12,0 \\
\hline
\end{tabular}

Source: Elaborated by the author

\section{Table 10}

Percentual reduction by Comparison 1, considering $\sigma_{\mathrm{Cp}}=2,0 \mathrm{MPa}$

\begin{tabular}{|c|c|c|c|c|c|c|c|c|c|c|c|}
\hline & & & $400 \mathrm{kN}$ & $500 \mathrm{kN}$ & $600 \mathrm{kN}$ & $700 \mathrm{kN}$ & $800 \mathrm{kN}$ & $900 \mathrm{kN}$ & $1000 \mathrm{kN}$ & $1100 \mathrm{kN}$ & $1200 \mathrm{kN}$ \\
\hline \multirow{6}{*}{$\begin{array}{c}\text { Column } \\
30 \times 30\end{array}$} & \multirow{2}{*}{ C 30} & $\rho=0,5 \%$ & - & - & 38,5 & 27,3 & 21,1 & 17,2 & 14,6 & 12,6 & - \\
\hline & & $\rho=1,5 \%$ & - & - & - & 37,8 & 26,4 & 20,3 & 16,5 & 13,9 & - \\
\hline & \multirow{2}{*}{ C 35} & $\rho=0,5 \%$ & - & - & 40,5 & 28,3 & 21,7 & 17,6 & 14,9 & 12,8 & 11,3 \\
\hline & & $\rho=1,5 \%$ & - & - & - & 41,2 & 28,0 & 21,2 & 17,1 & 14,3 & 12,3 \\
\hline & \multirow{2}{*}{ C 40} & $\rho=0,5 \%$ & - & - & 42,9 & 29,4 & 22,4 & 18,1 & 15,2 & 13,0 & 11,5 \\
\hline & & $\rho=1,5 \%$ & - & - & - & - & 31,3 & 23,4 & 18,8 & 15,6 & 13,4 \\
\hline \multirow{6}{*}{$\begin{array}{l}\text { Column } \\
40 \times 40\end{array}$} & \multirow{2}{*}{ C 30} & $\rho=0,5 \%$ & - & - & 50,0 & 34,0 & 25,8 & 20,7 & 17,3 & 14,9 & 13,1 \\
\hline & & $\rho=1,5 \%$ & - & - & - & - & 37,0 & 27,4 & 21,8 & 18,1 & 15,5 \\
\hline & \multirow{2}{*}{ C 35} & $\rho=0,5 \%$ & - & - & 51,6 & 34,0 & 25,4 & 20,3 & 16,8 & 14,4 & 12,6 \\
\hline & & $\rho=1,5 \%$ & - & - & - & - & 39,5 & 28,8 & 22,7 & 18,7 & 15,9 \\
\hline & \multirow{2}{*}{ C 40} & $\rho=0,5 \%$ & - & - & - & 37,8 & 27,9 & 22,1 & 18,3 & 15,6 & 13,6 \\
\hline & & $\rho=1,5 \%$ & - & - & - & - & 41,0 & 29,1 & 22,5 & 18,4 & 15,5 \\
\hline \multirow{6}{*}{$\begin{array}{l}\text { Column } \\
50 \times 50\end{array}$} & \multirow{2}{*}{ C 30} & $\rho=0,5 \%$ & - & - & - & 40,9 & 30,0 & 23,7 & 19,6 & 16,7 & 14,5 \\
\hline & & $\rho=1,5 \%$ & - & - & - & - & - & 34,5 & 26,8 & 21,8 & 18,4 \\
\hline & \multirow{2}{*}{ C 35} & $\rho=0,5 \%$ & - & - & - & 45,2 & 32,8 & 25,7 & 21,1 & 17,9 & 15,6 \\
\hline & & $\rho=1,5 \%$ & - & - & - & - & - & 37,3 & 28,4 & 22,9 & 19,2 \\
\hline & \multirow{2}{*}{ C 40} & $\rho=0,5 \%$ & - & - & - & 46,2 & 32,7 & 25,4 & 20,7 & 17,5 & 15,1 \\
\hline & & $\rho=1,5 \%$ & - & - & - & - & - & 38,3 & 28,6 & 22,8 & 18,9 \\
\hline
\end{tabular}


Table 11

Percentual reduction by Comparison 2, considering $\sigma_{\mathrm{cp}}=1,33 \mathrm{MPa}$

\begin{tabular}{|c|c|c|c|c|c|c|c|c|c|c|c|}
\hline & & & $400 \mathrm{kN}$ & $500 \mathrm{kN}$ & $600 \mathrm{kN}$ & $700 \mathrm{kN}$ & $800 \mathrm{kN}$ & $900 \mathrm{kN}$ & $1000 \mathrm{kN}$ & $1100 \mathrm{kN}$ & $1200 \mathrm{kN}$ \\
\hline \multirow{6}{*}{$\begin{array}{c}\text { Column } \\
30 \times 30\end{array}$} & \multirow{2}{*}{ C 30} & $\rho=0,5 \%$ & - & 71,4 & 52,3 & 41,7 & 36,8 & 32,6 & 30,6 & - & - \\
\hline & & $\rho=1,5 \%$ & - & - & - & 47,6 & 39,7 & 35,1 & 31,1 & - & - \\
\hline & \multirow{2}{*}{ C 35} & $\rho=0,5 \%$ & - & 76,9 & 54,8 & 43,1 & 37,8 & 33,3 & 31,1 & 28,7 & - \\
\hline & & $\rho=1,5 \%$ & - & - & - & 51,3 & 40,0 & 35,2 & 32,2 & 29,1 & - \\
\hline & \multirow{2}{*}{ C 40} & $\rho=0,5 \%$ & - & - & 55,0 & 42,9 & 37,5 & 34,1 & 30,8 & 29,2 & 27,2 \\
\hline & & $\rho=1,5 \%$ & - & - & - & 54,1 & 41,5 & 36,2 & 31,8 & 29,7 & 28,2 \\
\hline \multirow{6}{*}{$\begin{array}{l}\text { Column } \\
40 \times 40\end{array}$} & \multirow{2}{*}{ C 30} & $\rho=0,5 \%$ & - & - & 61,5 & 47,3 & 40,8 & 35,6 & 33,0 & 30,3 & 28,9 \\
\hline & & $\rho=1,5 \%$ & - & - & - & 60,0 & 45,1 & 38,8 & 34,9 & 31,3 & 29,6 \\
\hline & \multirow{2}{*}{ C 35} & $\rho=0,5 \%$ & - & - & 61,1 & 48,1 & 41,2 & 35,7 & 33,0 & 30,2 & 28,8 \\
\hline & & $\rho=1,5 \%$ & - & - & - & 62,5 & 47,9 & 40,6 & 35,0 & 32,3 & 29,5 \\
\hline & \multirow{2}{*}{ C 40} & $\rho=0,5 \%$ & - & - & 64,7 & 48,0 & 40,9 & 36,6 & 32,7 & 30,7 & 28,5 \\
\hline & & $\rho=1,5 \%$ & - & - & - & - & 51,1 & 41,0 & 36,4 & 32,3 & 30,3 \\
\hline \multirow{6}{*}{$\begin{array}{l}\text { Column } \\
50 \times 50\end{array}$} & \multirow{2}{*}{ C 30} & $\rho=0,5 \%$ & - & - & 70,6 & 54,0 & 43,9 & 39,0 & 35,7 & 32,5 & 30,8 \\
\hline & & $\rho=1,5 \%$ & - & - & - & - & 54,5 & 43,3 & 38,2 & 34,8 & 31,5 \\
\hline & \multirow{2}{*}{ C 35} & $\rho=0,5 \%$ & - & - & 74,2 & 55,3 & 44,4 & 39,2 & 35,8 & 32,4 & 30,7 \\
\hline & & $\rho=1,5 \%$ & - & - & - & - & 57,5 & 44,6 & 38,9 & 34,1 & 31,7 \\
\hline & \multirow{2}{*}{ C 40} & $\rho=0,5 \%$ & - & - & - & 57,8 & 45,9 & 40,3 & 35,5 & 33,0 & 31,2 \\
\hline & & $\rho=1,5 \%$ & - & - & - & - & 61,1 & 46,2 & 39,7 & 34,5 & 32,0 \\
\hline
\end{tabular}

\section{Table 12}

Percentual reduction by Comparison 2, considering $\sigma_{\mathrm{cp}}=2,0 \mathrm{MPa}$

\begin{tabular}{|c|c|c|c|c|c|c|c|c|c|c|c|}
\hline & & & $400 \mathrm{kN}$ & $500 \mathrm{kN}$ & $600 \mathrm{kN}$ & $700 \mathrm{kN}$ & $800 \mathrm{kN}$ & $900 \mathrm{kN}$ & $1000 \mathrm{kN}$ & $1100 \mathrm{kN}$ & $1200 \mathrm{kN}$ \\
\hline \multirow{6}{*}{$\begin{array}{l}\text { Column } \\
30 \times 30\end{array}$} & \multirow{2}{*}{ C 30} & $\rho=0,5 \%$ & - & - & 79,5 & 60,0 & 50,7 & 44,8 & 39,8 & - & - \\
\hline & & $\rho=1,5 \%$ & - & - & - & 78,4 & 58,5 & 47,8 & 42,4 & - & - \\
\hline & \multirow{2}{*}{ C 35} & $\rho=0,5 \%$ & - & - & 81,1 & 62,3 & 52,2 & 44,7 & 40,6 & 36,8 & - \\
\hline & & $\rho=1,5 \%$ & - & - & - & 82,4 & 60,0 & 50,0 & 42,7 & 38,8 & - \\
\hline & \multirow{2}{*}{ C 40} & $\rho=0,5 \%$ & - & - & 85,7 & 62,7 & 52,2 & 45,8 & 40,4 & 37,4 & 34,4 \\
\hline & & $\rho=1,5 \%$ & - & - & - & - & 64,6 & 51,6 & 43,8 & 39,6 & 36,6 \\
\hline \multirow{6}{*}{$\begin{array}{l}\text { Column } \\
40 \times 40\end{array}$} & \multirow{2}{*}{ C 30} & $\rho=0,5 \%$ & - & - & 94,1 & 70,0 & 56,1 & 48,8 & 43,9 & 39,5 & 36,9 \\
\hline & & $\rho=1,5 \%$ & - & - & - & - & 69,6 & 56,5 & 47,4 & 42,6 & 38,2 \\
\hline & \multirow{2}{*}{ C 35} & $\rho=0,5 \%$ & - & - & - & 72,3 & 57,1 & 49,4 & 43,2 & 39,6 & 37,0 \\
\hline & & $\rho=1,5 \%$ & - & - & - & - & 74,4 & 59,3 & 49,3 & 44,0 & 39,3 \\
\hline & \multirow{2}{*}{ C 40} & $\rho=0,5 \%$ & - & - & - & 75,6 & 59,0 & 50,6 & 44,1 & 40,4 & 36,8 \\
\hline & & $\rho=1,5 \%$ & - & - & - & - & 76,9 & 60,0 & 49,3 & 43,7 & 38,8 \\
\hline \multirow{6}{*}{$\begin{array}{l}\text { Column } \\
50 \times 50\end{array}$} & \multirow{2}{*}{ C 30} & $\rho=0,5 \%$ & - & - & - & 79,5 & 63,3 & 53,9 & 46,7 & 42,6 & 38,7 \\
\hline & & $\rho=1,5 \%$ & - & - & - & - & - & 65,5 & 54,9 & 48,3 & 42,7 \\
\hline & \multirow{2}{*}{ C 35} & $\rho=0,5 \%$ & - & - & - & 83,3 & 65,5 & 55,4 & 47,8 & 43,4 & 39,3 \\
\hline & & $\rho=1,5 \%$ & - & - & - & - & - & 68,6 & 56,7 & 48,2 & 43,4 \\
\hline & \multirow{2}{*}{ C 40} & $\rho=0,5 \%$ & - & - & - & 87,2 & 67,3 & 56,3 & 48,3 & 43,7 & 39,5 \\
\hline & & $\rho=1,5 \%$ & - & - & - & - & - & 72,3 & 57,1 & 49,4 & 43,2 \\
\hline
\end{tabular}




\section{Table 13}

Percentual reduction by Comparison 3, considering $\sigma_{\mathrm{cp}}=1,33 \mathrm{MPa}$

\begin{tabular}{|c|c|c|c|c|c|c|c|c|c|c|c|}
\hline & \multirow{3}{*}{$\begin{array}{c}400 \mathrm{kN} \\
-\end{array}$} & \multirow{3}{*}{$\begin{array}{c}500 \text { kN } \\
55,6\end{array}$} & \multirow{3}{*}{$\begin{array}{c}600 \mathrm{kN} \\
38,2\end{array}$} & \multirow{3}{*}{$\begin{array}{c}700 \text { kN } \\
30,0\end{array}$} & \multirow{3}{*}{$\begin{array}{c}800 \mathrm{kN} \\
27,3\end{array}$} & \multirow{3}{*}{$\begin{array}{c}900 \mathrm{kN} \\
24,4\end{array}$} & \multirow{3}{*}{$\begin{array}{c}1000 \mathrm{kN} \\
23,5\end{array}$} & \multirow{3}{*}{$\begin{array}{c}1100 \text { kN } \\
-\end{array}$} & \multirow{3}{*}{$\begin{array}{c}1200 \mathrm{kN} \\
-\end{array}$} \\
\hline & & & & & & & & & & & \\
\hline \multirow{6}{*}{$\begin{array}{c}\text { Column } \\
30 \times 30\end{array}$} & \multirow{2}{*}{ C 30} & $\rho=0,5 \%$ & & & & & & & & & \\
\hline & & $\rho=1,5 \%$ & - & - & - & 31,3 & 27,1 & 25,0 & 22,5 & - & - \\
\hline & \multirow{2}{*}{ C 35} & $\rho=0,5 \%$ & - & 62,5 & 40,6 & 31,3 & 28,1 & 25,0 & 24,0 & 22,3 & - \\
\hline & & $\rho=1,5 \%$ & - & - & - & 34,5 & 26,7 & 24,6 & 23,4 & 21,5 & - \\
\hline & \multirow{2}{*}{ C 40} & $\rho=0,5 \%$ & - & - & 40,0 & 30,4 & 27,4 & 25,6 & 23,4 & 22,7 & 21,4 \\
\hline & & $\rho=1,5 \%$ & - & - & - & 37,0 & 27,9 & 25,4 & 22,7 & 22,0 & 21,5 \\
\hline \multirow{6}{*}{$\begin{array}{l}\text { Column } \\
40 \times 40\end{array}$} & \multirow{2}{*}{ C 30} & $\rho=0,5 \%$ & - & - & 44,4 & 32,6 & 28,8 & 25,3 & 24,2 & 22,4 & 22,0 \\
\hline & & $\rho=1,5 \%$ & - & - & - & 41,7 & 30,0 & 26,8 & 25,0 & 22,7 & 22,1 \\
\hline & \multirow{2}{*}{ C 35} & $\rho=0,5 \%$ & - & - & 44,0 & 34,1 & 29,8 & 26,0 & 24,7 & 22,9 & 22,3 \\
\hline & & $\rho=1,5 \%$ & - & - & - & - & 30,6 & 26,9 & 23,5 & 22,6 & 21,0 \\
\hline & \multirow{2}{*}{ C 40} & $\rho=0,5 \%$ & - & - & 47,8 & 33,3 & 29,1 & 26,8 & 24,1 & 23,3 & 21,8 \\
\hline & & $\rho=1,5 \%$ & - & - & - & - & 33,3 & 26,5 & 24,6 & 22,2 & 21,6 \\
\hline \multirow{6}{*}{$\begin{array}{l}\text { Column } \\
50 \times 50\end{array}$} & \multirow{2}{*}{ C 30} & $\rho=0,5 \%$ & - & - & 52,4 & 37,8 & 30,2 & 27,5 & 25,9 & 23,8 & 23,1 \\
\hline & & $\rho=1,5 \%$ & - & - & - & - & 35,5 & 27,7 & 25,4 & 24,1 & 22,1 \\
\hline & \multirow{2}{*}{ C 35} & $\rho=0,5 \%$ & - & - & 55,6 & 38,2 & 30,0 & 27,3 & 25,6 & 23,5 & 22,8 \\
\hline & & $\rho=1,5 \%$ & - & - & - & - & 37,0 & 27,9 & 25,4 & 22,7 & 22,0 \\
\hline & \multirow{2}{*}{ C 40} & $\rho=0,5 \%$ & - & - & - & 40,6 & 31,3 & 28,1 & 25,0 & 24,0 & 23,2 \\
\hline & & $\rho=1,5 \%$ & - & - & - & - & - & 30,0 & 26,8 & 23,6 & 22,7 \\
\hline
\end{tabular}

Source: Elaborated by the author

\section{Table 14}

Percentual reduction by Comparison 3, considering $\sigma_{\mathrm{cp}}=2,0 \mathrm{MPa}$

\begin{tabular}{|c|c|c|c|c|c|c|c|c|c|c|c|}
\hline & & & $400 \mathrm{kN}$ & $500 \mathrm{kN}$ & $600 \mathrm{kN}$ & $700 \mathrm{kN}$ & 800 kN & $900 \mathrm{kN}$ & $1000 \mathrm{kN}$ & $1100 \mathrm{kN}$ & $1200 \mathrm{kN}$ \\
\hline \multirow{6}{*}{$\begin{array}{l}\text { Column } \\
30 \times 30\end{array}$} & \multirow{2}{*}{ C 30} & $\rho=0,5 \%$ & - & - & 66,7 & 45,0 & 37,5 & 33,3 & 29,5 & - & - \\
\hline & & $\rho=1,5 \%$ & - & - & - & 65,2 & 43,6 & 34,5 & 31,0 & - & - \\
\hline & \multirow{2}{*}{ C 35} & $\rho=0,5 \%$ & - & - & 68,2 & 47,4 & 38,9 & 32,9 & 30,2 & 27,5 & - \\
\hline & & $\rho=1,5 \%$ & - & - & - & 70,0 & 44,4 & 36,5 & 30,9 & 28,6 & - \\
\hline & \multirow{2}{*}{ C 40} & $\rho=0,5 \%$ & - & - & 75,0 & 47,2 & 38,5 & 33,8 & 29,8 & 28,0 & 25,9 \\
\hline & & $\rho=1,5 \%$ & - & - & - & - & 48,5 & 36,7 & 30,8 & 28,4 & 26,8 \\
\hline \multirow{6}{*}{$\begin{array}{l}\text { Column } \\
40 \times 40\end{array}$} & \multirow{2}{*}{ C 30} & $\rho=0,5 \%$ & - & - & 88,2 & 54,5 & 40,8 & 35,4 & 32,1 & 28,9 & 27,4 \\
\hline & & $\rho=1,5 \%$ & - & - & - & - & 51,7 & 40,0 & 32,8 & 29,9 & 26,9 \\
\hline & \multirow{2}{*}{ C 35} & $\rho=0,5 \%$ & - & - & - & 58,1 & 42,6 & 36,5 & 31,6 & 29,5 & 27,9 \\
\hline & & $\rho=1,5 \%$ & - & - & - & - & 57,7 & 42,9 & 34,5 & 31,1 & 27,8 \\
\hline & \multirow{2}{*}{ C 40} & $\rho=0,5 \%$ & - & - & - & 60,7 & 43,2 & 36,7 & 31,6 & 29,3 & 26,9 \\
\hline & & $\rho=1,5 \%$ & - & - & - & - & 60,9 & 43,6 & 34,5 & 31,0 & 27,6 \\
\hline \multirow{6}{*}{$\begin{array}{l}\text { Column } \\
50 \times 50\end{array}$} & \multirow{2}{*}{ C 30} & $\rho=0,5 \%$ & - & - & - & 65,4 & 47,6 & 39,7 & 33,8 & 31,1 & 28,3 \\
\hline & & $\rho=1,5 \%$ & - & - & - & - & - & 47,2 & 38,5 & 33,8 & 29,8 \\
\hline & \multirow{2}{*}{ C 35} & $\rho=0,5 \%$ & - & - & - & 69,6 & 48,7 & 40,0 & 33,8 & 31,0 & 28,2 \\
\hline & & $\rho=1,5 \%$ & - & - & - & - & - & 50,0 & 39,6 & 32,8 & 30,0 \\
\hline & \multirow{2}{*}{ C 40} & $\rho=0,5 \%$ & - & - & - & 76,2 & 51,4 & 41,5 & 34,8 & 31,8 & 28,7 \\
\hline & & $\rho=1,5 \%$ & - & - & - & - & - & 55,2 & 40,0 & 34,4 & 29,9 \\
\hline
\end{tabular}


Table 15

Percentual reduction by Comparison 4, considering $\sigma_{\mathrm{cp}}=1,33 \mathrm{MPa}$

\begin{tabular}{|c|c|c|c|c|c|c|c|c|c|c|c|}
\hline & \multirow{3}{*}{$\begin{array}{c}400 \mathrm{kN} \\
-\end{array}$} & \multirow{3}{*}{$\begin{array}{c}500 \text { kN } \\
87,9\end{array}$} & \multirow{3}{*}{$\begin{array}{c}600 \mathrm{kN} \\
75,9\end{array}$} & \multirow{3}{*}{$\begin{array}{c}700 \mathrm{kN} \\
67,9\end{array}$} & \multirow{3}{*}{$\frac{800 \mathrm{kN}}{-}$} & \multirow{3}{*}{$\frac{900 \mathrm{kN}}{-}$} & \multirow{3}{*}{$\begin{array}{c}1000 \mathrm{kN} \\
-\end{array}$} & \multirow{3}{*}{\begin{tabular}{|c|}
$1100 \mathrm{kN}$ \\
- \\
\end{tabular}} & \multirow{3}{*}{$\begin{array}{c}1200 \mathrm{kN} \\
-\end{array}$} \\
\hline & & & & & & & & & & & \\
\hline \multirow{6}{*}{$\begin{array}{l}\text { Column } \\
30 \times 30\end{array}$} & \multirow{2}{*}{ C 30} & $\rho=0,5 \%$ & & & & & & & & & \\
\hline & & $\rho=1,5 \%$ & - & - & - & 79,8 & - & - & - & - & - \\
\hline & \multirow{2}{*}{ C 35} & $\rho=0,5 \%$ & - & 90,5 & 77,4 & 68,6 & 63,5 & - & - & - & - \\
\hline & & $\rho=1,5 \%$ & - & - & - & 81,9 & 73,8 & - & - & - & - \\
\hline & \multirow{2}{*}{ C 40} & $\rho=0,5 \%$ & - & - & 77,8 & 68,9 & 63,4 & - & - & - & - \\
\hline & & $\rho=1,5 \%$ & - & - & - & 83,5 & 74,8 & - & - & - & - \\
\hline \multirow{6}{*}{$\begin{array}{l}\text { Column } \\
40 \times 40\end{array}$} & \multirow{2}{*}{ C 30} & $\rho=0,5 \%$ & - & - & 80,8 & 71,0 & 65,0 & 60,6 & - & - & - \\
\hline & & $\rho=1,5 \%$ & - & - & - & 86,0 & 76,7 & 71,1 & - & - & - \\
\hline & \multirow{2}{*}{ C 35} & $\rho=0,5 \%$ & - & - & 81,1 & 71,9 & 65,8 & 60,9 & - & - & - \\
\hline & & $\rho=1,5 \%$ & - & - & - & 87,5 & 78,6 & 72,5 & - & - & - \\
\hline & \multirow{2}{*}{ C 40} & $\rho=0,5 \%$ & - & - & 83,1 & 71,7 & 65,5 & 61,2 & 57,4 & - & - \\
\hline & & $\rho=1,5 \%$ & - & - & - & - & 80,5 & 73,1 & 68,4 & - & - \\
\hline \multirow{6}{*}{$\begin{array}{c}\text { Column } \\
50 \times 50\end{array}$} & \multirow{2}{*}{ C 30} & $\rho=0,5 \%$ & - & - & - & 74,7 & 67,0 & 62,4 & 59,1 & - & - \\
\hline & & $\rho=1,5 \%$ & - & - & - & - & 82,1 & 74,4 & 69,5 & - & - \\
\hline & \multirow{2}{*}{ C 35} & $\rho=0,5 \%$ & - & - & - & 75,6 & 67,3 & 62,5 & 59,1 & 56,1 & - \\
\hline & & $\rho=1,5 \%$ & - & - & - & - & 84,1 & 75,8 & 70,5 & 66,1 & - \\
\hline & \multirow{2}{*}{ C 40} & $\rho=0,5 \%$ & - & - & - & 76,8 & 68,0 & 62,9 & 58,6 & 56,0 & 54,3 \\
\hline & & $\rho=1,5 \%$ & - & - & - & - & 86,4 & 77,4 & 71,7 & 66,9 & 63,8 \\
\hline
\end{tabular}

\section{Table 16}

Percentual reduction by Comparison 4, considering $\sigma_{\mathrm{cp}}=2,0 \mathrm{MPa}$

\begin{tabular}{|c|c|c|c|c|c|c|c|c|c|c|c|}
\hline & & & $400 \mathrm{kN}$ & $500 \mathrm{kN}$ & $600 \mathrm{kN}$ & $700 \mathrm{kN}$ & $800 \mathrm{kN}$ & $900 \mathrm{kN}$ & $1000 \mathrm{kN}$ & $1100 \mathrm{kN}$ & $1200 \mathrm{kN}$ \\
\hline \multirow{6}{*}{$\begin{array}{c}\text { Column } \\
30 \times 30\end{array}$} & \multirow{2}{*}{ C 30} & $\rho=0,5 \%$ & - & - & 90,8 & 79,8 & - & - & - & - & - \\
\hline & & $\rho=1,5 \%$ & - & - & - & 92,7 & - & - & - & - & - \\
\hline & \multirow{2}{*}{ C 35} & $\rho=0,5 \%$ & - & - & 91,7 & 81,1 & 73,8 & - & - & - & - \\
\hline & & $\rho=1,5 \%$ & - & - & - & 94,3 & 84,1 & - & - & - & - \\
\hline & \multirow{2}{*}{ C 40} & $\rho=0,5 \%$ & - & - & 93,8 & 81,6 & 74,0 & - & - & - & - \\
\hline & & $\rho=1,5 \%$ & - & - & - & - & 86,2 & - & - & - & - \\
\hline \multirow{6}{*}{$\begin{array}{l}\text { Column } \\
40 \times 40\end{array}$} & \multirow{2}{*}{ C 30} & $\rho=0,5 \%$ & - & - & - & 85,0 & 75,8 & 70,4 & - & - & - \\
\hline & & $\rho=1,5 \%$ & - & - & - & - & 88,3 & 81,0 & - & - & - \\
\hline & \multirow{2}{*}{ C 35} & $\rho=0,5 \%$ & - & - & - & 86,5 & 76,9 & 71,0 & - & - & - \\
\hline & & $\rho=1,5 \%$ & - & - & - & - & 90,6 & 82,6 & - & - & - \\
\hline & \multirow{2}{*}{ C 40} & $\rho=0,5 \%$ & - & - & - & 88,0 & 77,9 & 71,6 & 66,5 & - & - \\
\hline & & $\rho=1,5 \%$ & - & - & - & - & 92,0 & 83,6 & 76,8 & - & - \\
\hline \multirow{6}{*}{$\begin{array}{l}\text { Column } \\
50 \times 50\end{array}$} & \multirow{2}{*}{ C 30} & $\rho=0,5 \%$ & - & - & - & - & 80,4 & 73,7 & 68,2 & - & - \\
\hline & & $\rho=1,5 \%$ & - & - & - & - & - & 85,7 & 79,2 & - & - \\
\hline & \multirow{2}{*}{ C 35} & $\rho=0,5 \%$ & - & - & - & - & 81,3 & 74,2 & 68,5 & 64,9 & - \\
\hline & & $\rho=1,5 \%$ & - & - & - & - & - & 87,5 & 80,5 & 74,9 & - \\
\hline & \multirow{2}{*}{ C 40} & $\rho=0,5 \%$ & - & - & - & - & 82,5 & 75,0 & 69,0 & 65,1 & 61,7 \\
\hline & & $\rho=1,5 \%$ & - & - & - & - & - & 89,5 & 81,4 & 75,9 & 71,3 \\
\hline
\end{tabular}




\section{Table 17}

Percentual reduction by Comparison 5, considering $\sigma_{\mathrm{cp}}=1,33 \mathrm{MPa}$

\begin{tabular}{|c|c|c|c|c|c|c|c|c|c|c|c|}
\hline & & & $400 \mathrm{kN}$ & $500 \mathrm{kN}$ & $600 \mathrm{kN}$ & $700 \mathrm{kN}$ & $800 \mathrm{kN}$ & $900 \mathrm{kN}$ & $1000 \mathrm{kN}$ & $1100 \mathrm{kN}$ & $1200 \mathrm{kN}$ \\
\hline \multirow{6}{*}{$\begin{array}{l}\text { Column } \\
30 \times 30\end{array}$} & \multirow{2}{*}{ C 30} & $\rho=0,5 \%$ & - & 57,6 & 49,4 & 45,0 & - & - & - & - & - \\
\hline & & $\rho=1,5 \%$ & - & - & 70,1 & 61,5 & - & - & - & - & - \\
\hline & \multirow{2}{*}{ C 35} & $\rho=0,5 \%$ & - & 58,7 & 50,0 & 44,8 & 41,3 & - & - & - & - \\
\hline & & $\rho=1,5 \%$ & - & - & 72,6 & 62,9 & 56,3 & - & - & - & - \\
\hline & \multirow{2}{*}{ C 40} & $\rho=0,5 \%$ & - & 60,0 & 50,6 & 45,6 & 41,5 & - & - & - & - \\
\hline & & $\rho=1,5 \%$ & - & - & 74,1 & 64,1 & 56,9 & - & - & - & - \\
\hline \multirow{6}{*}{$\begin{array}{l}\text { Column } \\
40 \times 40\end{array}$} & \multirow{2}{*}{ C 30} & $\rho=0,5 \%$ & - & - & 50,0 & 45,0 & 40,8 & 38,7 & - & - & - \\
\hline & & $\rho=1,5 \%$ & - & - & - & 65,0 & 57,5 & 52,8 & - & - & - \\
\hline & \multirow{2}{*}{ C 35} & $\rho=0,5 \%$ & - & - & 51,4 & 45,8 & 41,9 & 39,1 & - & - & - \\
\hline & & $\rho=1,5 \%$ & - & - & - & 66,7 & 59,0 & 53,6 & - & - & - \\
\hline & \multirow{2}{*}{ C 40} & $\rho=0,5 \%$ & - & - & 52,1 & 45,7 & 41,6 & 38,8 & 36,8 & - & - \\
\hline & & $\rho=1,5 \%$ & - & - & - & 68,5 & 60,2 & 54,5 & 50,3 & - & - \\
\hline \multirow{6}{*}{$\begin{array}{c}\text { Column } \\
50 \times 50\end{array}$} & \multirow{2}{*}{ C 30} & $\rho=0,5 \%$ & - & - & - & 45,1 & 41,1 & 38,3 & 36,4 & - & - \\
\hline & & $\rho=1,5 \%$ & - & - & - & 69,2 & 60,7 & 54,9 & 50,6 & - & - \\
\hline & \multirow{2}{*}{ C 35} & $\rho=0,5 \%$ & - & - & - & 45,3 & 41,1 & 38,3 & 36,2 & 35,1 & - \\
\hline & & $\rho=1,5 \%$ & - & - & - & 72,1 & 62,6 & 56,3 & 51,7 & 48,5 & - \\
\hline & \multirow{2}{*}{ C 40} & $\rho=0,5 \%$ & - & - & - & 45,1 & 40,8 & 37,9 & 35,9 & 34,3 & 33,5 \\
\hline & & $\rho=1,5 \%$ & - & - & - & - & 65,0 & 58,1 & 53,1 & 49,4 & 46,8 \\
\hline
\end{tabular}

\section{Table 18}

Percentual reduction by Comparison 5, considering $\sigma_{\mathrm{cp}}=2,0 \mathrm{MPa}$

\begin{tabular}{|c|c|c|c|c|c|c|c|c|c|c|c|}
\hline & & & $400 \mathrm{kN}$ & $500 \mathrm{kN}$ & $600 \mathrm{kN}$ & $700 \mathrm{kN}$ & 800 kN & $900 \mathrm{kN}$ & $1000 \mathrm{kN}$ & $1100 \mathrm{kN}$ & $1200 \mathrm{kN}$ \\
\hline \multirow{6}{*}{$\begin{array}{l}\text { Column } \\
30 \times 30\end{array}$} & \multirow{2}{*}{ C 30} & $\rho=0,5 \%$ & - & - & 55,2 & 49,5 & - & - & - & - & - \\
\hline & & $\rho=1,5 \%$ & - & - & 75,9 & 66,1 & - & - & - & - & - \\
\hline & \multirow{2}{*}{ C 35} & $\rho=0,5 \%$ & - & - & 56,0 & 50,0 & 45,2 & - & - & - & - \\
\hline & & $\rho=1,5 \%$ & - & - & - & 67,9 & 60,3 & - & - & - & - \\
\hline & \multirow{2}{*}{ C 40} & $\rho=0,5 \%$ & - & - & 56,8 & 50,5 & 45,5 & - & - & - & - \\
\hline & & $\rho=1,5 \%$ & - & - & - & 68,9 & 61,0 & - & - & - & - \\
\hline \multirow{6}{*}{$\begin{array}{l}\text { Column } \\
40 \times 40\end{array}$} & \multirow{2}{*}{ C 30} & $\rho=0,5 \%$ & - & - & - & 50,0 & 45,0 & 42,3 & - & - & - \\
\hline & & $\rho=1,5 \%$ & - & - & - & 70,0 & 61,7 & 56,3 & - & - & - \\
\hline & \multirow{2}{*}{ C 35} & $\rho=0,5 \%$ & - & - & - & 51,0 & 46,2 & 42,8 & - & - & - \\
\hline & & $\rho=1,5 \%$ & - & - & - & 71,9 & 63,2 & 57,2 & - & - & - \\
\hline & \multirow{2}{*}{ C 40} & $\rho=0,5 \%$ & - & - & - & 51,1 & 46,0 & 42,5 & 40,0 & - & - \\
\hline & & $\rho=1,5 \%$ & - & - & - & 75,0 & 65,5 & 59,0 & 54,2 & - & - \\
\hline \multirow{6}{*}{$\begin{array}{l}\text { Column } \\
50 \times 50\end{array}$} & \multirow{2}{*}{ C 30} & $\rho=0,5 \%$ & - & - & - & - & 46,4 & 42,9 & 40,3 & - & - \\
\hline & & $\rho=1,5 \%$ & - & - & - & - & 65,2 & 58,6 & 53,9 & - & - \\
\hline & \multirow{2}{*}{ C 35} & $\rho=0,5 \%$ & - & - & - & - & 45,8 & 42,2 & 39,6 & 38,0 & - \\
\hline & & $\rho=1,5 \%$ & - & - & - & - & 67,3 & 60,2 & 55,0 & 51,5 & - \\
\hline & \multirow{2}{*}{ C 40} & $\rho=0,5 \%$ & - & - & - & - & 46,6 & 42,7 & 40,0 & 38,0 & 36,7 \\
\hline & & $\rho=1,5 \%$ & - & - & - & - & 69,9 & 62,1 & 56,6 & 52,4 & 49,5 \\
\hline
\end{tabular}


Table 19

Percentual reduction by Comparison 6, considering $\sigma_{\mathrm{cP}}=1,33 \mathrm{MPa}$

\begin{tabular}{|c|c|c|c|c|c|c|c|c|c|c|c|}
\hline & \multirow{3}{*}{$\begin{array}{c}400 \mathrm{kN} \\
-\end{array}$} & \multirow{3}{*}{$\begin{array}{c}\mathbf{5 0 0} \mathbf{k N} \\
72,7 \\
\end{array}$} & \multirow{3}{*}{$\begin{array}{c}600 \mathrm{kN} \\
60,9\end{array}$} & \multirow{3}{*}{$\begin{array}{c}700 \mathrm{kN} \\
54,1 \\
\end{array}$} & \multirow{3}{*}{$\begin{array}{c}800 \mathrm{kN} \\
-\end{array}$} & \multirow{3}{*}{$\begin{array}{c}900 \mathrm{kN} \\
-\end{array}$} & \multirow{3}{*}{$\begin{array}{c}1000 \mathrm{kN} \\
- \\
\end{array}$} & \multirow{3}{*}{\begin{tabular}{|c|}
$1100 \mathrm{kN}$ \\
- \\
\end{tabular}} & \multirow{3}{*}{$\frac{1200 \mathrm{kN}}{-}$} \\
\hline & & & & & & & & & & & \\
\hline \multirow{6}{*}{$\begin{array}{l}\text { Column } \\
30 \times 30\end{array}$} & \multirow{2}{*}{ C 30} & $\rho=0,5 \%$ & & & & & & & & & \\
\hline & & $\rho=1,5 \%$ & - & - & - & 70,6 & - & - & - & - & - \\
\hline & \multirow{2}{*}{ C 35} & $\rho=0,5 \%$ & - & 74,6 & 61,9 & 54,3 & 49,2 & - & - & - & - \\
\hline & & $\rho=1,5 \%$ & - & - & - & 72,4 & 64,3 & - & - & - & - \\
\hline & \multirow{2}{*}{ C 40} & $\rho=0,5 \%$ & - & 76,7 & 63,0 & 55,3 & 49,6 & - & - & - & - \\
\hline & & $\rho=1,5 \%$ & - & - & - & 73,8 & 65,0 & - & - & - & - \\
\hline \multirow{6}{*}{$\begin{array}{l}\text { Column } \\
40 \times 40\end{array}$} & \multirow{2}{*}{ C 30} & $\rho=0,5 \%$ & - & - & 65,4 & 57,0 & 50,8 & 47,2 & - & - & - \\
\hline & & $\rho=1,5 \%$ & - & - & - & 76,0 & 66,7 & 60,6 & - & - & - \\
\hline & \multirow{2}{*}{ C 35} & $\rho=0,5 \%$ & - & - & 66,2 & 57,3 & 51,3 & 47,1 & - & - & - \\
\hline & & $\rho=1,5 \%$ & - & - & - & - & 69,2 & 62,3 & - & - & - \\
\hline & \multirow{2}{*}{ C 40} & $\rho=0,5 \%$ & - & - & 67,6 & 57,6 & 51,3 & 47,0 & 43,9 & - & - \\
\hline & & $\rho=1,5 \%$ & - & - & - & - & 70,8 & 63,4 & 58,1 & - & - \\
\hline \multirow{6}{*}{$\begin{array}{c}\text { Column } \\
50 \times 50\end{array}$} & \multirow{2}{*}{ C 30} & $\rho=0,5 \%$ & - & - & - & 59,3 & 52,7 & 48,1 & 44,8 & - & - \\
\hline & & $\rho=1,5 \%$ & - & - & - & - & 72,3 & 64,7 & 59,1 & - & - \\
\hline & \multirow{2}{*}{ C 35} & $\rho=0,5 \%$ & - & - & - & 60,5 & 53,3 & 48,4 & 45,0 & 42,7 & - \\
\hline & & $\rho=1,5 \%$ & - & - & - & - & 74,8 & 66,4 & 60,4 & 56,1 & - \\
\hline & \multirow{2}{*}{ C 40} & $\rho=0,5 \%$ & - & - & - & 61,0 & 53,4 & 48,4 & 44,8 & 42,2 & 40,4 \\
\hline & & $\rho=1,5 \%$ & - & - & - & - & - & 67,7 & 61,4 & 56,6 & 53,2 \\
\hline
\end{tabular}

\section{Table 20}

Percentual reduction by Comparison 6, considering $\sigma_{\mathrm{cp}}=2,0 \mathrm{MPa}$

\begin{tabular}{|c|c|c|c|c|c|c|c|c|c|c|c|}
\hline & & & $400 \mathrm{kN}$ & $500 \mathrm{kN}$ & $600 \mathrm{kN}$ & $700 \mathrm{kN}$ & $800 \mathrm{kN}$ & $900 \mathrm{kN}$ & $1000 \mathrm{kN}$ & $1100 \mathrm{kN}$ & $1200 \mathrm{kN}$ \\
\hline \multirow{6}{*}{$\begin{array}{l}\text { Column } \\
30 \times 30\end{array}$} & \multirow{2}{*}{ C 30} & $\rho=0,5 \%$ & - & - & 72,4 & 63,3 & - & - & - & - & - \\
\hline & & $\rho=1,5 \%$ & - & - & - & 78,9 & - & - & - & - & - \\
\hline & \multirow{2}{*}{ C 35} & $\rho=0,5 \%$ & - & - & 73,8 & 64,2 & 57,1 & - & - & - & - \\
\hline & & $\rho=1,5 \%$ & - & - & - & 81,1 & 71,4 & - & - & - & - \\
\hline & \multirow{2}{*}{ C 40} & $\rho=0,5 \%$ & - & - & 75,3 & 65,0 & 57,7 & - & - & - & - \\
\hline & & $\rho=1,5 \%$ & - & - & - & - & 73,2 & - & - & - & - \\
\hline \multirow{6}{*}{$\begin{array}{l}\text { Column } \\
40 \times 40\end{array}$} & \multirow{2}{*}{ C 30} & $\rho=0,5 \%$ & - & - & - & 67,0 & 59,2 & 54,2 & - & - & - \\
\hline & & $\rho=1,5 \%$ & - & - & - & - & 75,8 & 68,3 & - & - & - \\
\hline & \multirow{2}{*}{ C 35} & $\rho=0,5 \%$ & - & - & - & 67,7 & 59,8 & 54,3 & - & - & - \\
\hline & & $\rho=1,5 \%$ & - & - & - & - & 77,8 & 69,6 & - & - & - \\
\hline & \multirow{2}{*}{ C 40} & $\rho=0,5 \%$ & - & - & - & 69,6 & 61,1 & 55,2 & 51,0 & - & - \\
\hline & & $\rho=1,5 \%$ & - & - & - & - & 79,6 & 70,9 & 64,5 & - & - \\
\hline \multirow{6}{*}{$\begin{array}{l}\text { Column } \\
50 \times 50\end{array}$} & \multirow{2}{*}{ C 30} & $\rho=0,5 \%$ & - & - & - & - & 62,5 & 56,4 & 51,9 & - & - \\
\hline & & $\rho=1,5 \%$ & - & - & - & - & - & 72,9 & 66,2 & - & - \\
\hline & \multirow{2}{*}{ C 35} & $\rho=0,5 \%$ & - & - & - & - & 63,6 & 57,0 & 52,3 & 49,1 & - \\
\hline & & $\rho=1,5 \%$ & - & - & - & - & - & 75,0 & 67,8 & 62,6 & - \\
\hline & \multirow{2}{*}{ C 40} & $\rho=0,5 \%$ & - & - & - & - & 64,1 & 57,3 & 52,4 & 48,8 & 46,3 \\
\hline & & $\rho=1,5 \%$ & - & - & - & - & - & 76,6 & 69,0 & 63,3 & 59,0 \\
\hline
\end{tabular}


Analyzing the punching shear reinforcement calculated by using each design code, it is observed that if the prestress level is higher, less shear reinforcement is needed. Furthermore, the shear reinforcement are greatly influenced by flexural reinforcement ratio adopted, but varies slightly when changing the strength of concrete.

Analyzing the results shown in Tables 1 to 8 , it is noticed that the values found by Eurocode are the most economical, followed by NBR 6118:2014 and NBR 6118:2007. In the examples of the present study, $\mathrm{ACl} 318$ had the worst performance for all load levels and different variables in question.

In Leite Junior (2015) several comparative graphics, elaborated from Tables 9 to 20, show that for higher loads, the difference between the calculated punching shear reinforcement by the four standards tends to decrease, as well as the economy in relation to one and another. In addition, for each column section, the relation between the rules depends on the flexural reinforcement ratio, but varies slightly when changing concrete strength

NBR $6118: 2007$ provided a reduction of $5 \%$ to $25 \%$ in punching shear reinforcement by increasing the prestress. The formulation of this code considers, for load relief, only the tendons that pass $d / 2$ away from the column faces, so the results do not undergo significant change with this increase strain.

NBR 6118:2014 began to consider more influence of prestressing to calculate the shear strengh of slabs, since, besides the inclinated tendons component, also consider the value of $0,1 \sigma_{c p}$ as increasing the shear resistance. So, in general, it is observed a reduction percentage ranging from $12 \%$ to $40 \%$ by increasing the compression in the slab plane.

For the American standard, there is no change in the value of the calculated reinforcement varying the compression strain in the slab plane. This fact is explained by the limit imposed on the concrete strength, $0,17 \sqrt{f_{c}^{\prime}}$, when using shear reinforcement. Thus, it can be said that for the $\mathrm{ACl} 318-11$, increasing the prestress level has an influence only on the slabs without shear reinforcement, due to the factor of $0.3 \sigma_{\mathrm{cp}}$ adopted.

Since EC2 considers the inclined tendons that pass $2 d$ away from the column faces and also the factor $0,1 \sigma_{c p}$ as increasing the resistant strain, this code is more influenced by the prestressing level, providing reductions of approximately $20 \%$ to $86 \%$.

\subsection{Maximum loads without punching shear reinforcement}

Overall, the $\mathrm{ACl}$ has the boldest results when comparing the cases with lower flexural reinforcement ratio. Also the compression strength of concrete is quite limited by $\tau_{\mathrm{c}}$, imposed by the standard, providing no increase in the maximum load value reached from 35 $\mathrm{MPa}$, as can be seen in Table 21 .

EC2:2004 and NBR 6118:2014 are the less conservative standards in the cases of higher flexural reinforcement ratio and were slightly lower than the $\mathrm{ACl} 318$ results when these rates are low. NBR 6118:2007 generated the worst results of maximum loads when using low flexural reinforcement ratios. For high ratios, this code only overcomes ACI 318-11.

The maximum load supported by the slab increases when sections of the support columns are larger.

Increasing the compression strength of concrete provides more shear resistance to the slabs, except by using $\mathrm{ACl} 318$, as previously mentioned.

\section{Table 21}

Maximum factored shear force $(\mathrm{kN})$

\begin{tabular}{|c|c|c|c|c|c|c|c|c|c|c|c|}
\hline & & & \multicolumn{2}{|c|}{ NBR $6118: 2007$} & \multicolumn{2}{|c|}{ NBR 6118:2014 } & \multicolumn{2}{|c|}{$\mathrm{ACl} 318-11$} & \multicolumn{2}{|c|}{ EC2:2004 } & \multirow[b]{2}{*}{ Caso } \\
\hline & & & $\begin{array}{c}2 \\
\text { cabos } / m\end{array}$ & $\begin{array}{c}3 \\
\text { cabos/m }\end{array}$ & $\begin{array}{c}2 \\
\text { cabos } / m\end{array}$ & $\begin{array}{c}3 \\
\text { cabos } / \mathrm{m}\end{array}$ & $\begin{array}{c}2 \\
\text { cabos } / m\end{array}$ & $\begin{array}{c}3 \\
\text { cabos } / \mathrm{m}\end{array}$ & $\begin{array}{c}2 \\
\text { cabos } / m\end{array}$ & $\begin{array}{c}3 \\
\text { cabos } / \mathrm{m}\end{array}$ & \\
\hline \multirow{6}{*}{$\begin{array}{c}\text { Column } \\
30 \times 30\end{array}$} & \multirow{2}{*}{ C 30} & $\rho=0,5 \%$ & 403,0 & 435,0 & 465,0 & 527,0 & \multirow{2}{*}{465,0} & \multirow{2}{*}{529,0} & 472,0 & 545,0 & 1 \\
\hline & & $\rho=1,5 \%$ & 544,0 & 576,0 & 606,0 & 668,0 & & & 602,0 & 675,0 & 2 \\
\hline & \multirow{2}{*}{ C 35} & $\rho=0,5 \%$ & 420,0 & 451,0 & 482,0 & 544,0 & \multirow{2}{*}{484,0} & \multirow{2}{*}{548,0} & 487,0 & 560,0 & 3 \\
\hline & & $\rho=1,5 \%$ & 569,0 & 600,0 & 631,0 & 693,0 & & & 624,0 & 697,0 & 4 \\
\hline & \multirow{2}{*}{ C 40} & $\rho=0,5 \%$ & 435,0 & 467,0 & 497,0 & 559,0 & \multirow{2}{*}{484,0} & \multirow{2}{*}{548,0} & 502,0 & 574,0 & 5 \\
\hline & & $\rho=1,5 \%$ & 591,0 & 623,0 & 653,0 & 715,0 & & & 645,0 & 717,0 & 6 \\
\hline \multirow{6}{*}{$\begin{array}{l}\text { Column } \\
40 \times 40\end{array}$} & \multirow{2}{*}{ C 30} & $\rho=0,5 \%$ & 445,0 & 476,0 & 514,0 & 580,0 & \multirow{2}{*}{554,0} & \multirow{2}{*}{627,0} & 518,0 & 595,0 & 7 \\
\hline & & $\rho=1,5 \%$ & 604,0 & 635,0 & 674,0 & 740,0 & & & 665,0 & 742,0 & 8 \\
\hline & \multirow{2}{*}{ C 35} & $\rho=0,5 \%$ & 464,0 & 495,0 & 534,0 & 600,0 & \multirow{2}{*}{578,0} & \multirow{2}{*}{651,0} & 536,0 & 612,0 & 9 \\
\hline & & $\rho=1,5 \%$ & 632,0 & 663,0 & 701,5 & 768,0 & & & 691,0 & 767,0 & 10 \\
\hline & \multirow{2}{*}{ C 40} & $\rho=0,5 \%$ & 481,0 & 512,0 & 551,0 & 617,0 & \multirow{2}{*}{578,0} & \multirow{2}{*}{651,0} & 551,0 & 628,0 & 11 \\
\hline & & $\rho=1,5 \%$ & 657,0 & 688,0 & 726,0 & 792,0 & & & 714,0 & 791,0 & 12 \\
\hline \multirow{6}{*}{$\begin{array}{l}\text { Column } \\
50 \times 50\end{array}$} & \multirow{2}{*}{ C 30} & $\rho=0,5 \%$ & 486,0 & 517,0 & 564,0 & 634,0 & \multirow{2}{*}{644,0} & \multirow{2}{*}{726,0} & 564,0 & 645,0 & 13 \\
\hline & & $\rho=1,5 \%$ & 664,0 & 695,0 & 742,0 & 812,0 & & & 728,0 & 809,0 & 14 \\
\hline & \multirow{2}{*}{ C 35} & $\rho=0,5 \%$ & 507,0 & 538,0 & 585,0 & 655,0 & \multirow{2}{*}{671,0} & \multirow{2}{*}{753,0} & 584,0 & 664,0 & 15 \\
\hline & & $\rho=1,5 \%$ & 695,0 & 726,0 & 772,0 & 842,0 & & & 757,0 & 837,0 & 16 \\
\hline & \multirow{2}{*}{ C 40} & $\rho=0,5 \%$ & 526,0 & 558,0 & 605,0 & 674,0 & 6710 & 7530 & 601,0 & 682,0 & 17 \\
\hline & & $\rho=1,5 \%$ & 722,0 & 754,0 & 801,0 & 870,0 & $0 / 1,0$ & 153,0 & 782,0 & 863,0 & 18 \\
\hline
\end{tabular}




\section{Conclusions}

The analyzes show that Eurocode is a standard that generates more economic results of punching shear reinforcement, especially for higher loads, followed by NBR 6118:2014 and NBR 6118:2007. However, $\mathrm{ACl}$ is more conservative since the flexural reinforcement ratios do not affect the shear resistance and there are significant limitations relating to the strength of concrete. In general, for slabs with small overload, ACI 318-11 can generate an economic design, since the code can generate a result that dispenses the use of the punching shear reinforcement. EC2:2004 and NBR 6118:2014 have been following, with the NBR 6118:2007 generates the most conservative results. NBR 6118:2014 managed to bring the results of the Eurocode, even not considering the calculations the amount of tendons considered by EC2 to relieve vertical load. However, it can be said that the insertion of compressive strain in the slab plane has been an important factor in achieving less conservative results of punching shear reinforcements.

The compression in the slab plane could have already been studied and adopted before, as this has been done by Americans since the $60 \mathrm{~s}$, in a value three times higher than the European standard. Nevertheless, the latest revision of NBR was promising in the sense that the view may be changing aiming greater economy in the calculations, with designs increasingly accurate and efficient, especially for prestressed slabs.

It can be seen clearly that the results generated by the $\mathrm{ACl}$ are adversely affected by the fact that the contribution of the concrete $\left(\tau_{c}\right)$ and the critical section considered in the calculation of $V_{p}$ are quite limited. When there is the need of using punching shear reinforcement, the compression in the slab plane does not influence the calculations due to $\tau_{c}$ limitation. Thus, the prestressed slab is calculated just like reinforced concrete.

If it is necessary to use reinforcement punch, NBR 6118:2007 generates results slightly less conservative than $\mathrm{ACl}-318$, although the compression in the slab plane is not considered.

\section{References}

[1] AMERICAN CONCRETE INSTITUTE. ACI 318: Building Code Requirements for Structural Concrete. Detroit, 2011.

[2] ASSOCIAÇÃO BRASILEIRA DE NORMAS TÉCNICAS. NBR 6118: Projeto de estruturas de concreto - Procedimento. Rio de Janeiro, 2007.

[3] ASSOCIAÇÃO BRASILEIRA DE NORMAS TÉCNICAS. NBR 6118: Projeto de estruturas de concreto - Procedimento. Rio de Janeiro, 2014.

[4] EUROPEAN COMMITTEE FOR STANDARDIZATION. EUROCODE 2: Design of Concrete Structures - Part 1: General Rules and Rules for Building. Bruxelas, 2004

[5] LEITE JUNIOR, Jose Carlos Cirino, Dimensionamento à punção em apoios internos de lajes protendidas sem aderência. 2015. 157 f. Dissertação (Mestrado em Engenharia Civil), Universidade Federal do Espírito Santo, Vitória, 2015. 